\title{
3D multi-slab diffusion-weighted readout-segmented EPI with real-time cardiac-reordered k-space acquisition
}

Robert Frost ${ }^{1}$, Karla L. Miller ${ }^{1}$, Rob H. N. Tijssen ${ }^{2}$, David A. Porter ${ }^{3}$ and Peter Jezzard ${ }^{1}$

1. FMRIB Centre, Department of Clinical Neurosciences, University of Oxford, Oxford, UK

2. Department of Radiotherapy, University Medical Centre Utrecht, Utrecht, Netherlands

3. Siemens AG, Healthcare Sector, Erlangen, Germany

Word count: 6500

Running title: 3D multi-slab rs-EPI

Address for Correspondence:

Peter Jezzard, PhD

FMRIB Centre

John Radcliffe Hospital

Oxford

OX3 9DU

UK

Tel: +44 (0)1865 222727

Fax: +44 (0)1865 222717

Email: peterj@fmrib.ox.ac.uk 


\section{Abstract}

Purpose: To develop, implement and demonstrate a 3D extension of the readoutsegmented echo-planar imaging (rs-EPI) sequence for diffusion imaging.

Theory and Methods: Motion-induced phase artifacts were also simulated to test navigator corrections and a real-time reordering of the k-space acquisition relative to the cardiac cycle. The cardiac reordering strategy preferentially chooses readout segments closer to the centre of 3D k-space during diastole. Motion-induced phase artifacts were quantified by calculating the voxel-wise temporal variation in a set of repeated diffusion-weighted acquisitions. Based on the results of these simulations a 2D navigated multi-slab rs-EPI sequence with real-time cardiac reordering was implemented. The multi-slab implementation enables SNR-optimal TRs of 1-2 s. Results: Cardiac reordering was validated in simulations and in vivo using the multi-slab rs-EPI sequence. In comparisons with standard k-space acquisitions, cardiac reordering was shown to reduce the variability due to motion-induced phase artifacts by $30-50 \%$. High-resolution DTI data acquired with the cardiac-reordered multi-slab rs-EPI sequence is presented.

Conclusions: A 3D multi-slab rs-EPI sequence with cardiac reordering has been demonstrated in vivo and is shown to provide high-quality 3D diffusion-weighted data sets.

Keywords: diffusion MR; readout-segmented EPI; 3D diffusion-weighting; cardiac synchronisation; navigator correction; diffusion tensor imaging 


\section{Introduction}

Diffusion-weighted imaging has been an important tool in the diagnosis of stroke [1] and for diffusion tensor imaging (DTI) and white matter fibre tracking [2,3]. Images have typically been acquired with 2D multi-slice sequences using a single-shot echo-planar imaging (ss-EPI) readout [4]. This has proved a robust method for efficient acquisition of snapshot diffusion-weighted (DW) whole-brain images with $\sim 2 \mathrm{~mm}$ isotropic resolution, particularly for DTI where large numbers of diffusion-encoded volumes are required. However, in ss-EPI, higher resolution is tied to more off-resonance and $\mathrm{T}_{2}{ }^{*}$ blurring artifacts. Parallel imaging can be used to mitigate these artifacts [5-7] by reducing the effective echo-spacing and the readout duration by factors of 2-3, however the artifacts are worse at higher field. Resolution improvements and artifact reduction can be achieved by segmenting the k-space acquisition and using navigator techniques $[8,9]$ to account for the phase inconsistency between shots caused by motion during the diffusion-encoding gradients. Such approaches have been demonstrated in several sequences [10-14], including readout-segmented EPI (rs-EPI) [15-17], which de-couples resolution from distortion and blurring artifacts by segmenting k-space in the readout direction to allow a short echo-spacing and a short within-shot readout. In the method of Porter et al. [16] the navigator information is used to apply a 2D navigator correction [13] for mild non-linear phase corruption and also to identify shots with large (uncorrectable) motion-induced phase errors so that they can be re-acquired $[18,19]$. Scan times are lengthened compared to ss-EPI by the multi-shot nature of the acquisition and two acceleration strategies have been presented: 1) using partial Fourier techniques to reduce the number of repetition time (TR) periods [20] and 2) reducing TR with a simultaneous multi-slice acquisition [21].

Although in-plane spatial resolution challenges can be overcome, the resolution in the slice direction is usually not much higher than $\sim 1.5 \mathrm{~mm}$ due to the difficulties in generating sharp radio-frequency (RF) pulse profiles for thin slices. In addition, exciting a large number of thin slices to achieve full brain coverage results in long, non-optimum TR values. The low signal-to-noise ratio (SNR) in small voxels can also be problematic, especially 
when the signal is further attenuated due to diffusion weighting. For these reasons a 3D DW sequence offers a potential solution; 3D Fourier encoding within an excited volume could allow high-resolution, well-defined voxels in the slice-direction. 3D sequences can often have higher SNR, although whether this is achieved in practice depends crucially on the achievable timing of the 2D and 3D sequences being compared. Isotropic highresolution 3D DW data would facilitate identification of small infarcts and lesions and comparison with anatomical 3D images. The data would also be valuable for tractography because fewer fibre tract orientations will be present in smaller voxels thereby simplifying the DW signal $[22,23]$.

However, several challenges face 3D acquisitions that must first be addressed. In a multishot DW acquisition with volume excitation, the motion-induced phase variation in the through-slab direction is significant and must be accounted for. We refer to the k-space data that is used to form the high-resolution image as "imaging" data and the k-space data that is used to correct the imaging data as "navigator" data. An extension to 3D of the nonlinear navigator correction in 2D rs-EPI would require 3D navigator data, which is difficult to realise. The resolution of the imaging and navigator data is limited by the long time required to cover even a small 3D volume, which reduces the efficiency of the sequence. Also, the imaging data has susceptibility artifacts in the slow phase-encoding direction and point-spread function (PSF) problems caused by amplitude and phase modulations in the final k-space matrix that is formed from the multiple shots. Some previous sequences have avoided multi-shot correction by using methods such as driven equilibrium diffusion preparation [24], acquiring a restricted volume [25-27], imaging in areas where motion phase errors are small [25,28], and by cardiac gating the acquisition [25,29,30]. 3D steadystate sequences have been explored with pseudo-self-navigation using a 3D navigator compiled from multiple TRs [31] and with an additional rigid-body correction at each TR [32]. To date those methods still exhibit residual motion corruption that would compromise diffusion measures, particularly for diffusion-encoding in the superior-inferior direction where brain pulsation is most pronounced and causes the most severe artifacts [13]. 
In this study, we consider alternatives for navigator-corrected 3D rs-EPI data. We used a realistic simulation environment to compare two acquisition schemes that acquire 2D or 3D trajectories within 3D k-space for each shot of high-resolution imaging data. In addition, motion-induced phase artifacts were simulated, including a reordering of the k-space acquisition with respect to the cardiac cycle $[13,33]$. Previous work has synchronised the acquisition to the cardiac and respiratory cycles to reduce ghosting artifacts in structural imaging [34,35] and temporal fluctuations in 3D segmented-acquisition functional MRI (fMRI) [33,36]. Cardiac synchronisation has also been used in segmented DW acquisitions to improve the accuracy of the "refocusing" navigator correction by enforcing a smoother variation of k-space phase and amplitude between interleaves in the combined data [13]. Shots close to the centre of 3D k-space are preferentially acquired in diastole to obtain cleaner data and the effect on image quality is tested in simulations and experiments.

The implemented scheme uses 2D imaging and navigator data in a novel 3D Fourierencoded multi-slab extension of the rs-EPI sequence. Multi-slab acquisitions can approach SNR optimal TRs of 1-2 s and, as we shall see below, motion artifacts in thin slabs are well approximated by a 2D navigator $[37,38]$. The rs-EPI method offers reduced susceptibility artifacts and $\mathrm{T}_{2}$ * blurring compared to similar multi-slab methods which encode a full 2D kspace partition at each excitation [38], at the expense of a longer scan time per volume.

\section{Theory}

\section{Assessment of 3D Readout-Segmented EPI Acquisition Schemes}

Two k-space trajectories for extension of rs-EPI to 3D encoded acquisition are shown in Fig. 1a. Both trajectories build on rs-EPI by acquiring 2D planes within the full 3D k-space (where a given $\mathrm{k}_{\mathrm{z}}$ plane is achieved through addition of an appropriate $\mathrm{k}_{\mathrm{z}}$ phase-encoding gradient). One trajectory (Fig. 1a, top row) is based on single 2D $\mathrm{k}_{\mathrm{z}}$ planes for both imaging and navigator data. We refer to the separate parts of this trajectory as $\mathrm{IM}_{2 \mathrm{D}}$ and $\mathrm{NAV}_{2 \mathrm{D}}$, respectively, and the combined trajectory as $\mathrm{IM}_{2 \mathrm{D}}+\mathrm{NAV} \mathrm{V}_{2 \mathrm{D}}$. The second trajectory (Fig. $1 \mathrm{a}$, 
bottom row) acquires several contiguous $\mathrm{k}_{\mathrm{z}}$ planes for both the imaging and navigator data. This trajectory is based on the single-shot echo-volumar imaging (EVI) technique [39,40], in which spatial encoding can be applied in all three directions during a single readout. We refer to the separate parts of this trajectory as $\mathrm{IM}_{3 \mathrm{D}}$ and $\mathrm{NAV}_{3 \mathrm{D}}$, respectively, and the combined trajectory as $\mathrm{IM}_{3 \mathrm{D}}+\mathrm{NAV} \mathrm{V}_{3 \mathrm{D}}$. The $\mathrm{IM}_{2 \mathrm{D}}+\mathrm{NAV} \mathrm{V}_{2 \mathrm{D}}$ method can only achieve a $2 \mathrm{D}$ navigator correction, while $\mathrm{IM}_{3 \mathrm{D}}+\mathrm{NAV}_{3 \mathrm{D}}$ is aimed at "proper" $3 \mathrm{D}$ navigation. For both schemes, as in rs-EPI, the imaging trajectories ( IM $_{2 D}$ and IM $\mathrm{M}_{3 \mathrm{D}}$ ) acquire different (2D or 3D) segments of k-space from one TR to the next (indicated by the arrows in Fig. 1a) so that the high-resolution k-space matrix is filled, while the navigator remains at the centre of k-space for all shots.

The imaging and navigator acquisitions are independent so, for example, $\mathrm{IM}_{2 \mathrm{D}}$ data could be acquired with $N A V_{3 D}$ data etc. From a motion correction perspective, acquiring $\mathrm{IM}_{3 \mathrm{D}}$ and $\mathrm{NAV}_{3 \mathrm{D}}$ data at contiguous $\mathrm{k}$-space locations (i.e., at adjacent $\mathrm{k}_{\mathrm{z}}$ planes) would allow an extension to 3D of the non-linear navigator correction in 2D rs-EPI. Alternatively, $\mathrm{IM}_{2 \mathrm{D}}$ data could be acquired with $\mathrm{NAV}_{3 \mathrm{D}}$ data so that phase information in $\mathrm{z}$ could be used to correct phase offsets and through-plane ramps in the 2D imaging data.

\section{Motion-Induced Phase Simulations}

In order to test acquisition and reconstruction strategies in the context of a 3D DW sequence, a simulation environment was implemented in Matlab (Mathworks, Natick, MA) for evaluation of motion-induced diffusion phase artifacts. Translations and rotations of the head are rigid-body movements and have been shown to impart constant and linear phase offsets, respectively $[8,9]$. Deformation of brain tissue during the cardiac cycle is a nonrigid motion that generates a non-linear spatial phase variation across the brain when it occurs during the diffusion-encoding gradients [13]. This motion has been measured with MR techniques [41-43] and manifests as if the brainstem is pulled downwards in a funnelshaped motion during systole thus reshaping the medial and inferior parts of the brain.

Wirestam et al. [44] described a model for the temporal and spatial variation of the $\mathrm{z}$ 
component of brain tissue velocity, $\mathrm{v}_{\mathrm{z}}(\mathrm{x}, \mathrm{y}, \mathrm{z}, \mathrm{t})$, based on experimental data from previous studies $[41,45]$ and used it to calculate signal loss caused by intravoxel dephasing when diffusion encoding is orientated in the $\mathrm{z}$ direction. In this study we use the model presented by Wirestam et al. to generate the expected phase maps for diffusion encoding along z so that multi-shot DW acquisitions can be simulated. This diffusion encoding direction has been shown to be the most problematic for diffusion phase artifacts $[13,31]$ so acquisition and reconstruction strategies can be tested in the worst-case scenario.

The equations for the temporal and spatial variation of velocity used in the simulations were taken from Ref. [44]. Temporal variation in velocity is given by Eq. 1:

$$
v_{z}(0,0,0, t)=c_{1}+c_{2} e^{-c_{3}\left(t_{0}-t\right)^{2}}
$$

where $c_{1}=-0.21 \mathrm{~mm} / \mathrm{s}, \mathrm{c}_{2}=2.4 \mathrm{~mm} / \mathrm{s}$ and $\mathrm{c}_{3}=295 \mathrm{~s}^{-2}$ and the origin is in the centre of an axial slice at the level of the pons. The parameter $t_{0}$ is the time from the start of the pulse sequence simulation to the maximum brain tissue velocity. The spatial velocity profile is given by Eq. 2:

$$
v_{z}(x, y, z, t)=v_{z}(0,0,0, t) e^{-a_{1}\left(x^{2}+y^{2}\right)-a_{2} z^{4.31}}
$$

where $\mathrm{a}_{1}=0.0022 \mathrm{~mm}^{-2} \mathrm{a}_{2}=2.3 \times 10^{-8} \mathrm{~mm}^{-4.31}$. Examples of the temporal and spatial variation of velocity are plotted in Supplementary Fig. 1.

Using this model for $\mathrm{v}_{\mathrm{z}}(\mathbf{r}, \mathrm{t})$, the displacement in $\mathrm{z}$ since the start of the pulse sequence, $d_{z}(r, t)$, could be calculated at each time step. Hence maps of the motion-induced phase imparted by the Stejskal-Tanner diffusion gradients, $\varphi(\mathbf{r})$, could be generated with Equations 3 and 4.

$$
d_{z}(\mathbf{r}, t)=\int_{0}^{t} v_{z}\left(\mathbf{r}, t^{\prime}\right) d t^{\prime}
$$




$$
\phi(\mathbf{r}, t)=\gamma \int_{0}^{T E} d_{z}(\mathbf{r}, t) G(t) d t
$$

\section{Cardiac Reordering of k-Space Shots}

The main motivation for these motion-phase simulations was to investigate a reordering of the k-space acquisition with respect to the cardiac cycle. We apply the cardiac reordering technique to a multi-shot DW acquisition with two compatible aims: 1) acquiring shots that are close to each other in k-space at similar points in the cardiac cycle (so that they have similar phase corruption) to ensure smooth phase and amplitude modulation, and 2) acquiring clean data (in diastole) close to the centre of k-space. As discussed in Ref. [13], locally consistent phase-corruption allows a more accurate k-space deconvolution in the refocusing correction. Also, a perturbation of the low spatial frequency components of the image due to phase error is more damaging to the overall image quality than when the same error is applied to the higher spatial frequencies.

Cardiac gating and previous reordering schemes affect the acquisition time and introduce a variable TR. Tijssen et al. were able to use a fixed TR by implementing an adaptive GRAPPA reconstruction to fill in parts of k-space that could not be acquired at the optimum time [33]. In the following simulations, the closest available shot to the corresponding cardiac phase is thus acquired. This ensures that data can be acquired continuously during the cardiac cycle using a constant TR. Shots acquired during systole that have severe phase corruption could potentially be re-acquired. On-line re-acquisition was simulated, but was not implemented in this study because it would have required modification of the on-line reconstruction code that was beyond the scope of this work. However, this could be done as future work.

\section{SNR Efficiency}

In order to achieve near-optimal SNR within a target scan time, the TR can be chosen to maximise an approximation of the SNR efficiency, the ratio signal $/ \sqrt{\mathrm{TR}}$. This analysis 
assumes all factors that affect noise (bandwidth, number of slices, number of averages etc.) are held constant and does not consider whether this TR is achievable with a particular sequence. The choice of TR is also influenced by the other considerations such as the scan time per volume and sensitivity to motion. SNR efficiency for a spin-echo sequence simulated at $3 \mathrm{~T}$ is plotted as a function of TR in Supplementary Fig. 2 using literature values for white matter tissue $T_{1}=1000 \mathrm{~ms}, T_{2}=75 \mathrm{~ms}[46,47]$ and echo time $=70 \mathrm{~ms}$. Numerical simulation of the Bloch equations was used to calculate the spin-echo signal. From the plot in Supplementary Fig. 2 a TR of $\sim 1.5 \mathrm{~s}$ will result in optimum SNR efficiency [48]. Using this TR would compromise whole brain coverage with standard 2D multi-slice techniques and would require prohibitively long scan times with 3D single-slab imaging. However, interleaved 3D multi-slab imaging can operate close to this TR regime without compromising slice coverage or volume scan times. A simultaneous multi-slab acquisition [49] can be used to reduce TR further and could be particularly beneficial in highresolution protocols.

\section{Methods}

\section{Simulations}

Point-Spread Functions of Acquisition Schemes

We investigated the effects of time-varying signal phase and amplitude on the trajectory PSF using simulations based around a $0.6 \times 0.6 \times 0.6 \mathrm{~mm}$ structural image and a $1 \times 1 \times 2.5 \mathrm{~mm}$ $\mathrm{B}_{0}$ field map, both originally acquired in a representative healthy human volunteer. The field map was upsampled (interpolated by zero padding in the Fourier domain) and the structural scan downsampled so that each voxel of the structural image contained $5 \times 5 \times 5$ field map values in order to simulate dephasing of signal due to field gradients. The simulation was performed by starting with a $120 \times 120 \times 10 \mathrm{k}$-space matrix of the downsampled structural image and then selecting regions of this matrix to acquire in different shots according to the $\mathrm{IM}_{2 \mathrm{D}}$ or $\mathrm{IM}_{3 \mathrm{D}}$ acquisition scheme. 
The effect of $\mathrm{B}_{0}$ inhomogeneity was incorporated into the simulated $\mathrm{k}$-space data in the following way. Each k-space point (from the downsampled structural image) was placed in its corresponding location in a $120 \times 120 \times 10$ matrix of zeros and Fourier transformed to image space where it was given the inhomogeneity phase (from the upsampled field map) corresponding to its position in the readout. The timestep resolution between simulated kspace points was $2 \mu$ s and a linear accrual of phase was used during the readout such that the phase was zero at the centre of the readout (at the spin-echo centre). Using the field map, the off-resonance phase could be calculated at each time (or k-space) point in the readout. Each sub-set of k-space covered by one shot of the $\mathrm{IM}_{2 \mathrm{D}}$ or $\mathrm{IM}_{3 \mathrm{D}}$ trajectory was simulated using this procedure. All the shots were combined to form the complete k-space.

The phase and the time-evolving image space mean signal (i.e., the signal at the centre of kspace during the spin-echo) were used to generate estimates of the spatial PSF. The complex signal was mapped onto the corresponding k-space locations for each readout and the multiple readouts were combined before the PSF was calculated by Fourier transformation.

Motion-Induced Phase

The simulations used TE $=70 \mathrm{~ms}$ and the diffusion gradient timings were duration $\delta=20$ $\mathrm{ms}$, diffusion time $\Delta=37 \mathrm{~ms}$. A diffusion gradient amplitude of $33.4 \mathrm{mT} / \mathrm{m}$ was used for a $\mathrm{b}$-value of $1000 \mathrm{~s} / \mathrm{mm}^{2}$. Phase maps at different readout shots were generated for random points in the cardiac cycle by varying the parameter $\mathrm{t}_{0}$ in Eq. 1 between -100 and $100 \mathrm{~ms}$. This approach samples a $200 \mathrm{~ms}$ interval around the systolic region and was chosen to simulate an extreme case of cardiac deformation. Simulations were performed using a numerical Shepp-Logan phantom and at each shot Rician noise was added to the magnitude image [50]. The phase maps for each motion state were generated with the model for velocity described above and were saved so that different acquisition and reconstruction strategies could be compared. 
The motion-induced phase error is different at each shot or TR so a multi-shot DW acquisition was simulated by applying the phase at each shot by complex multiplication in image space before transforming the phase-corrected image back to k-space and selecting the part of k-space sampled by the readout trajectory. The final multi-shot k-space was a combination of all these subsets of k-space. The choice of acquisition schemes to investigate in relation to the phase errors was dependent on the results of the PSF study. The imaging data constituted a readout segment (i.e., limited $\mathrm{k}_{\mathrm{x}}$ extent, full $\mathrm{k}_{\mathrm{y}}$ extent) from a single $\mathrm{k}_{\mathrm{z}}$ partition (trajectory $\mathrm{IM}_{2 \mathrm{D}}$ ) and the navigator could either be 1) a 2D fully sampled $\mathrm{k}_{\mathrm{y}}$ readout segment at the centre of 3D k-space (trajectory $\mathrm{NAV}_{2 \mathrm{D}}$ ) or 2) a 3D stack of $\mathrm{k}_{\mathrm{z}}$ planes around the centre of $\mathrm{k}_{\mathrm{z}}$ with limited extent in $\mathrm{k}_{\mathrm{x}}$ and $\mathrm{k}_{\mathrm{y}}$ (trajectory $\mathrm{NAV}_{3 \mathrm{D}}$ ). Using the terms defined in Fig. 1a and above, the two simulated acquisitions were $\mathrm{IM}_{2 \mathrm{D}}+\mathrm{NAV} \mathrm{V}_{2 \mathrm{D}}$ and $\mathrm{IM}_{2 \mathrm{D}}+\mathrm{NAV}$ 3D. The navigator correction was performed in $2 \mathrm{D}$ or $3 \mathrm{D}$ as appropriate i.e. 2D refocusing correction for the $\mathrm{NAV}_{2 \mathrm{D}}$ data and $3 \mathrm{D}$ refocusing for $\mathrm{NAV}_{3 \mathrm{D}}$ data. Re-acquisition of motion corrupted segments [18], which is used in the 2D sequence, was simulated with $20 \%$ of scan time dedicated to re-acquiring the k-space shots with the worst motion corruption. The re-acquisition was weighted towards shots at the centre of 3D k-space.

The reconstructions compared in simulations were as follows: no correction, 2D navigation with $\mathrm{NAV}_{2 \mathrm{D}}$ data and 3D navigation with $\mathrm{NAV}_{3 \mathrm{D}}$ data. Cardiac reordering of shots and reacquisition was simulated with all reconstructions. The voxel size in the simulations was $2 \times 2 \times 2 \mathrm{~mm}$ so $8 \mathrm{k}_{\mathrm{z}}$ phase-encodes corresponds to a slab thickness of $16 \mathrm{~mm}$. Artifact performance of the reconstructions was assessed via the quality of the resultant images or via maps of the coefficient of variation ( $\mathrm{CoV}$ ) over a set of 6 repeated simulations. The $\mathrm{CoV}$ maps demonstrate how variable the images are and were computed in each voxel by dividing the temporal standard deviation in signal by the temporal mean signal. The mean and standard deviation of the $\mathrm{CoV}$ in the high pulsatility region (velocity at peak systole $>0.5 \mathrm{~mm} / \mathrm{s}$ ) were calculated.

\section{In vivo}


Based on the simulation results (see below), we used a $\mathrm{IM}_{2 \mathrm{D}}+\mathrm{NAV} \mathrm{V}_{2 \mathrm{D}}$ version of 3D rs-EPI with 2D navigator correction and cardiac reordering of shots, which we demonstrate by acquiring data with $\mathrm{b}=1000 \mathrm{~s} / \mathrm{mm}^{2}$ and with a slab thickness $\sim 16 \mathrm{~mm}$. The 2D navigator correction of 2D planes of $\mathrm{k}_{\mathrm{x}}, \mathrm{k}_{\mathrm{y}}$ imaging data at 3D phase-encoded $\mathrm{k}_{\mathrm{z}}$ locations (IM $\left.\mathrm{M}_{2 \mathrm{D}}\right)$ was compared against theoretical predictions with in vivo experiments. The 2D navigator is always the central readout segment at the central $k_{z}$ partition, i.e. the $\mathrm{NAV}_{2 \mathrm{D}}$ scheme. Additionally, the cardiac reordering of shots was compared to a standard (sequential) kspace acquisition scheme.

Implementation

A standard 2D rs-EPI sequence [16] was modified to include slice gradient pre- and rephasing either side of the imaging echo so that readout segments could be $\mathrm{k}_{\mathrm{z}}$ phaseencoded ( $\left(\mathrm{M}_{2 \mathrm{D}} \mathrm{scheme}\right)$ and the navigator sampled the central $\mathrm{k}_{\mathrm{x}}, \mathrm{k}_{\mathrm{z}}$ readout segment ( $\mathrm{NAV}_{2 \mathrm{D}}$ scheme). Using the abbreviations introduced above, this sequence was a $\mathrm{IM}_{2 \mathrm{D}}+\mathrm{NAV} \mathrm{V}_{2 \mathrm{D}}$ acquisition. The modified sequence is shown in Fig. $1 \mathrm{~b}$.

Cardiac reordering of k-space shots was implemented in the sequence so that the decision on which shot to acquire at each excitation was made at sequence run time. The shot choice was determined by the position in the cardiac cycle, which was estimated using pulse oximeter information. Unlike cardiac gating, this reordering scheme does not alter the sequence timing. The cardiac phase, $\varphi_{c}$, was calculated by dividing the time since the cardiac trigger, $t_{\text {trig, }}$ measured with a pulse oximeter placed on the index finger (a $200 \mathrm{~ms}$ delay time between the finger and the head was determined empirically), by the mean cardiac cycle duration, $\mathrm{T}_{\mathrm{RR}}$ (from the previous 10 cardiac cycles), as shown in Eq. 5.

$$
\phi_{c}=t_{\text {trig }} / T_{R R} \text { (5) }
$$

A cardiac phase of 0.4 - 0.6 was assumed to correspond to diastole and therefore to be the optimum time to acquire data. An algorithm (the Matlab version is provided in the 
supplementary material) was used to determine the $\mathrm{k}_{\mathrm{z}}$ and $\mathrm{k}_{\mathrm{x}}$ shot indices $\left(\mathrm{n}_{\mathrm{z}}\right.$ and $\mathrm{n}_{\mathrm{x}}$, respectively).

When $\varphi_{\mathrm{c}}=0.5$, the algorithm chooses the central $\mathrm{k}_{\mathrm{x}}, \mathrm{k}_{\mathrm{z}}$ readout segment. For example, with $8 \mathrm{k}_{\mathrm{z}}$ phase-encodes $(\mathrm{Nz}=8)$ and 5 readout segments $(\mathrm{Nx}=5)$ the optimum shot is $\mathrm{nz}=5$, $\mathrm{nx}=3$. As $\varphi_{\mathrm{c}}$ tends towards 0 or 1 the chosen shot moves toward the edges of 3D k-space. As well as acquiring central $\mathrm{k}$-space during the quiet portion of the cardiac cycle, this scheme also ensures that adjacent segments will have similar values of cardiac phase and therefore that the residual phase-corruption after navigator correction will change smoothly through k-space [13]. If the chosen shot had already been acquired, the closest unacquired shot was selected. With this method, as the k-space matrix fills up, the selected shots usually become further away from the optimum position. Therefore, a measured cardiac phase between 0.4 and 0.6 is altered to 0.5 with the intention that shots near the centre of k-space are acquired early so that the outer k-space shots are acquired later, when they are more likely to be acquired with a sub-optimal cardiac phase. Logs of cardiac phase and selected shots were created to verify this strategy of ensuring early acquisition of shots at the centre of kspace. In a multi-slab acquisition, lists of shots were created for each slab and the shots were selected independently in each slab with the method described above. The objective of the scheme is to acquire cleaner data at the centre of k-space where corrupt data is more likely to result in severe signal loss artifacts. Although re-acquisition was simulated, it was not implemented in the sequence because it required modification of the on-line reconstruction code to accept 3D phase-encoded data rather than 2D multi-slice data, which was beyond the scope of this work.

To achieve contiguous slices at the interface between neighbouring slabs in a multi-slab acquisition a combination of slab overlap, $\mathrm{k}_{\mathrm{z}}$ oversampling [51,52], and separate slab concatenations was used [30]. The approaches used for slab overlap and $\mathrm{k}_{\mathrm{z}}$ oversampling are illustrated in Fig. 1c. To reduce saturation between slabs, all readout segments and $\mathrm{k}_{\mathrm{z}}$ phase-encodes were acquired in separate concatenations for odd and even slabs. This increased the acquisition time by a factor of two but reduced artifacts at interfaces between slabs when scanning with TR $\sim 1 \mathrm{~s}$. RF pulses designed with the SLR algorithm [53] were 
used to create a flatter profile of signal across the slab and narrower transition bands. The excitation pulse had time-bandwidth product (TBWP) of 18 and a duration of $5 \mathrm{~ms}$ and the refocusing pulses had TBWPs of 8 and $10 \mathrm{~ms}$ durations.

Images were reconstructed off-line in Matlab from raw scanner data. The rs-EPI reconstruction used was similar to that described in previous work [20] but with modifications for the 3D phase-encoded data. The full pipeline was: phase-correction on a slab-specific basis using a pre-scan at $\mathrm{k}_{\mathrm{y}}=\mathrm{k}_{\mathrm{z}}=0$ and regridding to account for the sinusoidal readout gradient waveform; GRAPPA reconstruction of missing $\mathrm{k}_{\mathrm{y}}$ lines [54]; 2D navigator correction of readout segments at all $\mathrm{k}_{\mathrm{x}}, \mathrm{k}_{\mathrm{z}}$ locations with a navigator readout segment at the central $\mathrm{k}_{\mathrm{x}}, \mathrm{k}_{\mathrm{z}}$ location; compilation of a 3D k-space matrix after discarding the extra $\mathrm{k}_{\mathrm{x}}$ columns at the edges of each readout segment; 3D Fourier transform and combination of coil data with sum of squares reconstruction. GRAPPA under-sampling of $\mathrm{k}_{\mathrm{z}}$ phase-encodes was not possible due to the small z FOV of each slab, which would lead to large g-factor-related losses when trying to un-alias voxels a maximum of $8 \mathrm{~mm}$ apart (16 $\mathrm{mm}$ slab with factor 2 under-sampling). In partial Fourier encoded data, POCS partial Fourier reconstruction $[20,55]$ was used to estimate the missing portion of k-space.

Data Acquisition and Processing

Data were acquired in five healthy volunteers with a MAGNETOM Verio 3 T scanner (Siemens Healthcare, Erlangen, Germany) under an approved technical development ethics protocol. A pulse oximeter was used to determine the cardiac phase as described above. The diffusion preparation module was a modified Stejskal-Tanner pair [56] to allow a shorter TE. As a consequence of having two refocusing pulses in the sequence, the excitation angle used was the Ernst angle given by $\cos ^{-1}\left(\mathrm{e}^{-\mathrm{TR} / \mathrm{T} 1}\right)$, rather than the spin-echo Ernst angle $\left(\pi-\cos ^{-1}\left(\mathrm{e}^{-\mathrm{TR} / \mathrm{T} 1}\right)\right)$ for a spin-echo sequence with a single refocusing pulse. Nonbrain tissue was removed from the images using the FSL Brain Extraction Tool [57].

\section{Variability in Repeated Diffusion Acquisitions}


Data were acquired from five subjects, with and without cardiac reordering of shots to verify the improvement in image quality predicted by simulation. One $\mathrm{T}_{2}$-weighted volume without diffusion weighting and six DW volumes with $\mathrm{b}=1000 \mathrm{~s} / \mathrm{mm}^{2}$ encoding in the $\mathrm{z}$ direction (the encoding direction that is most sensitive to motion artifacts) were acquired with a 12-channel head coil. The scan times of the 8 and $16 \mathrm{k}_{\mathrm{z}}$ phase-encode acquisitions were 4:45 min and 9:25 min, respectively, and each acquisition was repeated with and without cardiac reordering. The acquisition parameters are given in Table 1. The repeated DW volumes were used to assess the variability of the images. Voxel-wise maps of coefficient of variation (CoV) were computed by dividing the temporal standard deviation in signal by the temporal mean signal.

\section{Multi-Slab DTI}

DTI data with isotropic resolutions of 1.3 and $1.1 \mathrm{~mm}$ were acquired in two subjects. The $1.1 \mathrm{~mm}$ protocol attempted to minimize slab-joining artifacts with higher TBWP pulses and acquired all slabs in one TR concatenation. Both protocols acquired three $\mathrm{T}_{2}$-weighted volumes and $20 \mathrm{DW}$ volumes with orthogonal $\mathrm{b}=1000 \mathrm{~s} / \mathrm{mm}^{2}$ encoding with a 32-channel head coil. The acquisition parameters are given in Table 1. A correction was also applied to the $1.1 \mathrm{~mm}$ data to reduce signal saturation at the boundary as follows. A mean diffusionweighted image was generated to estimate the signal variation in the slice direction. This image was brain-extracted and the mean signal in each slice was calculated so that a mean slab profile could be estimated from all the slabs. For each slab in the mean diffusionweighted image, the least-squares fit amplitude of this mean slab profile was used to derive a magnitude correction that accounts for the drop in signal between slabs. The same weighting was applied to all images in the DTI protocol. This correction is shown in Supplementary Fig. 3. Affine registration (12 degrees of freedom) of volumes to the first $\mathrm{T}_{2^{-}}$ weighted volumes and fitting of data to the diffusion tensor was performed using FSL [58]. BEDPOSTX [59] was used to generate probability density distributions on two crossing fibre orientations at each voxel. A white matter mask was generated by thresholding the FA map at 0.25 and the voxels that supported a 2 nd fibre were determined by thresholding the BEDPOSTX 2nd fibre volume fraction at 0.05 . 


\section{Results}

\section{Simulations}

Point-Spread Functions of Acquisition Schemes

The phase and amplitude variation with time of the image space mean signal (i.e., the signal at the centre of k-space) during the spin-echo are shown in the left-hand column of Fig. 2. This spin-echo signal includes dephasing effects due to the sub-sampling in the simulation. In the plot of phase variation, the maximum phase error of $\pi / 2$ at the extremes of the spinecho readout is calculated from an average value of inhomogeneity across the brain. In the right-hand column of Fig. 2, these phase and amplitude variations are mapped onto one shot of a IM $\mathrm{IM}_{3 \mathrm{D}}$ scheme (shown in Fig. 1a) which samples $5 \mathrm{k}_{\mathrm{z}}$ planes in one shot. Note that each $\mathrm{k}_{\mathrm{y}}$ point represents one phase encode line (along $\mathrm{k}_{\mathrm{x}}$ ). This demonstrates the amplitude and phase discontinuities in the $\mathrm{k}_{\mathrm{z}}$ direction caused by the jump to a new $\mathrm{k}_{\mathrm{z}}$ plane at the initial $\mathrm{k}_{\mathrm{y}}$ position, in this case after the readout of $24 \mathrm{k}_{\mathrm{y}}$ lines.

When k-space is sampled in multiple shots with this $\mathrm{IM}_{3 \mathrm{D}}$ scheme, the amplitude variation in Fig. 2 (lower right panel) will be repeated through k-space. The $\mathrm{k}_{\mathrm{x}}$ dimension was split into 5 readout segments in both the $\mathrm{IM}_{2 \mathrm{D}}$ and $\mathrm{IM}_{3 \mathrm{D}}$ schemes. In the $\mathrm{IM}_{3 \mathrm{D}}$ scheme these segments were divided into $5 \mathrm{k}_{\mathrm{z}}$ planes so 5 shots were required to fill the y resolution. The number of $\mathrm{k}_{\mathrm{y}}$ shots multiplied by the number of $\mathrm{k}_{\mathrm{z}}$ shots is the same for both schemes so they have the same acquisition time. The results of a $\mathrm{IM}_{2 \mathrm{D}}$ scheme are shown in Fig. 3a and the results of a IM 3 s scheme in Fig. $3 b$.

These results suggest that the PSF in the $\mathrm{IM}_{3 \mathrm{D}}$ scheme introduces aliasing artifacts (indicated by the off-centre peaks along $\mathrm{z}$ and $\mathrm{y}$ ) as well as some blurring. Blurring is exhibited by a broadening of the main PSF peak from a pure delta function. These artifacts are most prominent along $\mathrm{z}$, where signal from alternating slices will alias. There are no 
PSF problems in the simulations with the IM2D scheme apart from relatively minor blurring along $y$.

In light of these simulations, it was decided that the $\mathrm{IM}_{2 \mathrm{D}}$ trajectory was most promising and that this was the strategy to develop further. Motion phase artifacts were simulated with $\mathrm{IM}_{2 \mathrm{D}}+\mathrm{NAV}_{2 \mathrm{D}}$ navigator correction and a $\mathrm{IM}_{2 \mathrm{D}}+\mathrm{NAV}_{2 \mathrm{D}}$ scheme was implemented in the sequence. A navigator correction of $\mathrm{IM}_{2 \mathrm{D}}+\mathrm{NAV}_{3 \mathrm{D}}$ data was simulated but not implemented, with further work required on this topic.

Motion-Induced Phase

Images of the phantom used for motion-induced phase simulations are shown for reference in Fig. 4a. Figures $4 \mathrm{~b}$ and $\mathrm{c}$ show that the 2D and 3D navigator corrections have similar performance, with slightly better image quality in the 3D correction. However, the 2D correction was chosen to be the best candidate for implementation (i.e., scheme $\mathrm{IM}_{2 \mathrm{D}}+\mathrm{NAV} \mathrm{V}_{2 \mathrm{D}}$ ) due to the close relationship of the sequence and the reconstruction to the standard 2D rs-EPI sequence. Figures $4 \mathrm{~b}$ and $\mathrm{c}$ also demonstrate major improvements in image quality with cardiac reordering of the k-space acquisition. The results show that after cardiac reordering and navigator correction, smaller improvements in image quality can be achieved by re-acquiring the shots with the worst motion corruption [18] in an extension of the current 2D rs-EPI implementation [16]. The simulations suggest (data not shown) that a navigator consisting of either a readout segment at the central $\mathrm{k}_{\mathrm{z}}$ partition $\left(\mathrm{NAV}_{2 \mathrm{D}}\right)$ or a readout segment split into a stack of $\mathrm{k}_{\mathrm{z}}$ partitions with low $\mathrm{k}_{\mathrm{y}}$ resolution $\left(\mathrm{NAV}_{3 \mathrm{D}}\right)$ at the centre of 3D k-space can be used to judge motion corruption.

\section{In vivo}

The cardiac cycle durations observed in the volunteers were in the range $600-1200 \mathrm{~ms}$. Images and variability results from a representative subject are shown in Fig. 5a for 8 and $162 \mathrm{~mm}$ slices. These results demonstrate the successful 2D navigation and the clear reduction of image artifacts with cardiac reordering of k-space shots. The largest 
improvements (high to low $\mathrm{CoV}$ ) are in the centre of the brain (where the non-rigid deformation is concentrated) and some surrounding white matter structures. In the cardiac-reordered data, the only remaining regions of high $\mathrm{CoV}$ are in low signal areas (e.g. CSF). In the $32 \mathrm{~mm}$ slab data the remaining CoV increases compared to the $16 \mathrm{~mm}$ slab data. In the raw DW images, the effect of reordering is clearer in the $32 \mathrm{~mm}$ slab data which exhibit artifactual low signal in the central areas of the brain in the sequential k-space acquisition. This signal is restored in the cardiac-reordered data. The mean $\mathrm{CoV}$ within the ROIs shown in Fig. $5 \mathrm{~b}$ was calculated so that quantitative results could be compared. A summary of simulation and experiment results in five subjects for $b=1000 \mathrm{~s} / \mathrm{mm}^{2}$ diffusion weighting in $\mathrm{z}$ with the 2D navigator correction (without re-acquisition) is shown in Table 2. For $8 \mathrm{k}_{\mathrm{z}}$ phase-encodes, a consistent $30-40 \%$ improvement with cardiac reordering was observed in the simulations and experiments, and the improvement increased with more $\mathrm{k}_{\mathrm{z}}$ phase-encodes/thicker slabs.

$1.1 \mathrm{~mm}$ multi-slab $\mathrm{T}_{2}$ - and diffusion-weighted images with a correction for the slab-joining artifacts are shown in Figs. 6a and b with evidence of some residual slab-joining artifacts. Fractional anisotropy (FA) and mean diffusivity (MD) maps are shown in Figs. 6c and d with less obvious artifacts after fitting between $\mathrm{T}_{2}$ - and diffusion-weighted data. This artifact reduction is expected since the artifacts should have equal weighting in both the $\mathrm{T}_{2}$ and diffusion-weighted images. Using the $1.3 \mathrm{~mm}$ multi-slab data, a demonstration of the resolution of the DTI data is shown in Fig. 7 and crossing fibre results are shown in Fig. 8. The data shows cerebellar projections in Fig. 7a and cortical anisotropy in Fig. 7b. Figure 8 shows voxels supporting a second fibre population and crossing fibre results in the parietal lobe that highlight the contrast in the data. $43 \%$ of voxels in the white matter mask supported a second fibre population. $1.1 \mathrm{~mm}$ images without slab-join correction and 1.1 mm DTI results are provided for comparison in the supplementary material.

\section{Discussion}

This study has demonstrated through simulation and experiment that 2D navigation can be 
used in thin-slab 3D rs-EPI DW acquisitions and that reordering the k-space acquisition with respect to the cardiac cycle results in clear improvements in image quality. Acquisition of 2D imaging $\left(\mathrm{IM}_{2 \mathrm{D}}\right)$ data was chosen based on PSF simulations, which suggested that 3D imaging ( $\left.\mathrm{IM}_{3 \mathrm{D}}\right)$ schemes would suffer from substantial aliasing artifacts. Simulations of motion phase artifacts using a model for brain velocity suggested 2D and 3D corrections $\left(\mathrm{IM}_{2 \mathrm{D}}+\mathrm{NAV} \mathrm{V}_{2 \mathrm{D}}\right.$ and $\left.\mathrm{IM}_{2 \mathrm{D}}+\mathrm{NAV}_{3 \mathrm{D}}\right)$ were possible and that cardiac reordering of the k-space acquisition and re-acquisition of motion corrupted shots were beneficial. Further work on the 3D navigator reconstruction (scheme $\mathrm{IM}_{2 \mathrm{D}}+\mathrm{NAV}_{3 \mathrm{D}}$ ) of experimental data is required. Modification of the current 2D on-line reconstruction code to accept 3D phase-encoded data would enable re-acquisition, which is expected to improve image quality further.

Predictions made in the simulation environment were confirmed experimentally with successful 2D navigator correction and reduction in variability in repeated acquisitions when diffusion weighting in $\mathrm{z}$ (the most problematic encoding direction for diffusionweighted artifacts). As expected, there was an increase in artifacts with stronger diffusion encoding and in thicker slabs. Stronger diffusion encoding creates a more complex phase variation across the brain with more phase wraps and in a larger slab there is more scope for phase difference in the slab direction. When there is more complicated phase variation in $\mathrm{z}$ the 2D or 3D navigator data becomes less effective at resolving and removing the phase corruption. Therefore the data contain residual phase corruption which causes signal loss and ghosting artifacts due to destructive phase interference as well as discontinuities and periodicity in the k-space signal [13]. The phase variation at each shot is unpredictable so in repeated acquisitions these artifacts will be different, leading to increasing variation in the temporal standard deviation maps.

In quantitative analyses of the reduction of image artifacts, a 30-50\% reduction in variability was observed with cardiac reordering, in both simulations and experiments. Ideally, all shots would be acquired as cleanly as possible with no data acquisition during systole (which is not the case in the current reordering scheme). However, as mentioned above, cardiac gating approaches only allow 2-3 slabs per R-R interval resulting in variable 
and longer acquisition times, which are already long in 3D rs-EPI due to the segmentation of $\mathrm{k}$-space in $\mathrm{k}_{\mathrm{x}}$ and $\mathrm{k}_{\mathrm{z}}$. With cardiac reordering, clean data is acquired close to the centre of $\mathrm{k}$-space and phase corruption is locally consistent. The reordering scheme does not lengthen the acquisition time and can be implemented using real-time calculations based on physiological monitoring of the cardiac phase at each shot in a similar fashion to a recent reordering scheme applied to fMRI [33]. The reordering strategy should also be applicable to other segmented 3D DW sequences. Heart-rate variability could interfere with the cardiac reordering scheme but in the healthy volunteers scanned to date it has not been a significant problem. Implementing a re-acquisition of the shots with worst phase corruption is expected to improve residual levels of phase corruption caused by shots that were not acquired at the optimal time. The adaptive GRAPPA method proposed by Tijssen et al. [33] for filling in corrupt or poorly ordered shots is not possible in this multi-slab case due to lack of coil coverage in the slab direction. Bulk motion of the slab during the encoding could also cause substantial signal variations. After inspection of the scans on cooperative volunteers in this study, it does not appear to affect the consistency of the navigator correction. Motion correction between k-space segments [38] should be investigated as future work.

Artifacts at the interfaces between slabs are currently evident in the multi-slab data. Further optimisation of the slab overlap, RF pulses and post-processing correction is required to generate clean, isotropic data. Recent work in a 3D DW scan (TR $\sim 5$ s) with 2D navigator correction used high time-bandwith product $10 \mathrm{~ms}$ refocusing pulses and regridding of slabs with a slab profile weighting to generate data without visible slab crosstalk effects [38]. Although a TR $=1-2 \mathrm{~s}$ is optimal for SNR efficiency, the increased $\mathrm{T}_{1}$ saturation effects that cause slab-joining artifacts may necessitate a longer TR. In situations where the minimum TR is limited by a large number of slabs (higher slice resolution acquisitions would require more than 16 slabs used in this study) simultaneous multi-slab acceleration could be used [49,60]. The slice-GRAPPA method [61] can be used to un-alias multiplexed k-space data. Also, we have used partial Fourier encoding to reduce the number of readout segments required in the $\mathrm{k}_{\mathrm{x}}$ dimension [20]. 
Despite that fact that the volume scan times limit the number of diffusion directions, we were able to achieve excellent quality DTI. In particular, our data demonstrate a surprisingly robust estimate of crossing fibres, given the very low angular resolution, with BEDPOSTX supporting the evidence for a second fibre in a large fraction (43\%) of white matter voxels. These results must be interpreted with caution, since direction-correlated image artifacts could yield apparent complex fibre structure; however, the spatial distribution of these second fibre voxels is consistent with the established observation of few second fibres in major, homogeneous tracts (e.g., corpus callosum) and more complex fibre architecture close to cortex and in known crossing regions (e.g., the centrum semiovale) $[59,62]$. This likely reflects the unusually high SNR in the 3D acquisitions, as well as the high resolution and low level of artifact. The methods proposed above for faster 3D volume acquisition would translate directly into a greater number of directions, and likely improved modeling of complex fibre architecture.

\section{Conclusions}

A 3D rs-EPI multi-slab sequence for acquisition of true isotropic voxels with an SNRoptimal TR has been demonstrated. An acquisition trajectory for 3D imaging was chosen based on simulations of PSF artifacts. Using this trajectory, a method involving 2D navigator correction and cardiac reordering of the k-space acquisition was tested in simulation and was implemented in vivo. This showed good quality DW data with low levels of motion-phase artifact. A quantitative analysis of simulated and experimental data

suggests a $30-50 \%$ reduction in diffusion phase-induced variability instabilities when using real time cardiac reordering of k-space shots compared to a sequential acquisition. Scan time reduction through simultaneous multi-slab acceleration and optimisation of the number readout segments will be the subject of future work, along with implementation of 3D navigation and navigator-based re-acquisition.

\section{Acknowledgements}


The authors would like to thank Thorsten Feiweier (Siemens Healthcare, Erlangen) for the diffusion preparation used in this study and the UK Medical Research Council for funding.

\section{References}

1. Moseley ME, Cohen Y, Mintorovitch J, Chileuitt L, Shimizu H, Kucharczyk J, Wendland MF, Weinstein PR. Early detection of regional cerebral ischemia in cats: comparison of diffusion- and T2-weighted MRI and spectroscopy. Magn Reson Med 1990;14:330-346.

2. Basser PJ, Mattiello J, LeBihan D. MR diffusion tensor spectroscopy and imaging. Biophys J 1994;66:259-267.

3. Mori S, Crain BJ, Chacko VP, van Zijl PC. Three-dimensional tracking of axonal projections in the brain by magnetic resonance imaging. Ann Neurol 1999;45:265-269.

4. Turner R, Le Bihan D, Chesnick AS. Echo-planar imaging of diffusion and perfusion. Magn Reson Med 1991;19:247-253.

5. Griswold MA, Jakob PM, Chen Q, Goldfarb JW, Manning WJ, Edelman RR, Sodickson DK. Resolution enhancement in single-shot imaging using simultaneous acquisition of spatial harmonics (SMASH). Magn Reson Med 1999;41:1236-1245.

6. Heidemann RM, Griswold MA, Porter DA, Kiefer B, Nittka M, Wang J, Haase A, Jakob PM. Minimizing distortions and blurring in diffusion weighted single shot EPI using high performance gradients in combination with parallel imaging. In: Proceedings of the 9th Annual Meeting of ISMRM, 2001 (abstract 169).

7. Bammer R, Keeling SL, Augustin M, Pruessmann KP, Wolf R, Stollberger R, Hartung HP, Fazekas F. Improved diffusion-weighted single-shot echo-planar imaging (EPI) in stroke using sensitivity encoding (SENSE). Magn Reson Med 2001;46:548-554.

8. Ordidge RJ, Helpern JA, Qing ZX, Knight RA, Nagesh V. Correction of motional artifacts in diffusion-weighted MR images using navigator echoes. Magn Reson Imaging 1994;12:455-460.

9. Anderson AW, Gore JC. Analysis and correction of motion artifacts in diffusion weighted imaging. Magn Reson Med 1994;32:379-387.

10. Butts K, Pauly J, de Crespigny A, Moseley M. Isotropic diffusion-weighted and spiralnavigated interleaved EPI for routine imaging of acute stroke. Magn Reson Med 1997;38:741749. 
11. Atkinson D, Porter DA, Hill DL, Calamante F, Connelly A. Sampling and reconstruction effects due to motion in diffusion-weighted interleaved echo planar imaging. Magn Reson Med 2000;44:101-109.

12. Pipe JG, Farthing VG, Forbes KP. Multishot diffusion-weighted FSE using PROPELLER MRI. Magn Reson Med 2002;47:42-52.

13. Miller KL, Pauly JM. Nonlinear phase correction for navigated diffusion imaging. Magn Reson Med 2003;50:343-353.

14. Liu C, Bammer R, Kim D, Moseley ME. Self-navigated interleaved spiral (SNAILS): application to high-resolution diffusion tensor imaging. Magn Reson Med 2004;52:1388-1396.

15. Robson MD, Anderson AW, Gore JC. Diffusion-weighted multiple shot echo planar imaging of humans without navigation. Magn Reson Med 1997;38:82-88.

16. Porter DA, Heidemann RM. High resolution diffusion-weighted imaging using readoutsegmented echo-planar imaging, parallel imaging and a two-dimensional navigator-based reacquisition. Magn Reson Med 2009;62:468-475.

17. Holdsworth SJ, Skare S, Newbould RD, Bammer R. Robust GRAPPA-accelerated diffusionweighted readout-segmented (RS)-EPI. Magn Reson Med 2009;62:1629-1640.

18. Nguyen Q, Clemence M, Ordidge RJ. The use of intelligent re-acquisition to reduce scan time in MRI degraded by motion. In: Proceedings of the 6th Annual Meeting of ISMRM, 1998 (abstract 134).

19. Porter DA. 2D-navigator-based re-acquisition for motion artefact suppression in multi-shot, diffusion-weighted imaging. In: Proceedings of the 14th Annual Meeting of ISMRM, 2006 (abstract 1047).

20. Frost R, Porter DA, Miller KL, Jezzard P. Implementation and assessment of diffusionweighted partial Fourier readout-segmented echo-planar imaging. Magn Reson Med 2012;68:441-451.

21. Frost R, Porter DA, Douaud G, Jezzard P, Miller KL. Reduction of diffusion-weighted readout-segmented EPI scan time using a blipped-CAIPI modification. In: Proceedings of the 20th Annual Meeting of ISMRM, 2012 (abstract 116).

22. Frank LR. Anisotropy in high angular resolution diffusion-weighted MRI. Magn Reson Med 2001;45:935-939.

23. Frank LR. Characterization of anisotropy in high angular resolution diffusion-weighted MRI. Magn Reson Med 2002;47:1083-1099. 
24. Jeong E, Kim S, Parker DL. High-resolution diffusion-weighted 3D MRI, using diffusionweighted driven-equilibrium (DW-DE) and multishot segmented 3D-SSFP without navigator echoes. Magn Reson Med 2003;50:821-829.

25. Golay X, Jiang H, van Zij1 PCM, Mori S. High-resolution isotropic 3D diffusion tensor imaging of the human brain. Magn Reson Med 2002;47:837-843.

26. Wang J, Deichmann R, Turner R, Ordidge R. 3D DT-MRI using a reduced-FOV approach and saturation pulses. Magn Reson Med 2004;51:853-857.

27. Jeong E, Kim S, Kholmovski EG, Parker DL. High-resolution DTI of a localized volume using 3D single-shot diffusion-weighted STimulated echo-planar imaging (3D ss-DWSTEPI). Magn Reson Med 2006;56:1173-1181.

28. Miller KL, Hargreaves BA, Gold GE, Pauly JM. Steady-state diffusion-weighted imaging of in vivo knee cartilage. Magn Reson Med 2004;51:394-398.

29. Jung Y, Samsonov AA, Block WF, Lazar M, Lu A, Liu J, Alexander AL. 3D diffusion tensor MRI with isotropic resolution using a steady-state radial acquisition. J Magn Reson Imaging 2009;29:1175-1184.

30. Van AT, Hernando D, Sutton BP. Motion-induced phase error estimation and correction in 3D diffusion tensor imaging. IEEE Trans Med Imaging 2011;30:1933-1940.

31. McNab JA, Gallichan D, Miller KL. 3D steady-state diffusion-weighted imaging with trajectory using radially batched internal navigator echoes (TURBINE). Magn Reson Med 2010;63:235-242.

32. O'Halloran RL, Aksoy M, Van AT, Bammer R. 3D isotropic high-resolution diffusionweighted MRI of the whole brain with a motion-corrected steady-state free precession sequence. Magn Reson Med 2013;70:466-478.

33. Tijssen RHN, Okell TW, Miller KL. Real-time cardiac synchronization with fixed volume frame rate for reducing physiological instabilities in 3D FMRI. Neuroimage 2011;57:1364-1375.

34. Bailes DR, Gilderdale DJ, Bydder GM, Collins AG, Firmin DN. Respiratory ordered phase encoding (ROPE): a method for reducing respiratory motion artefacts in MR imaging. J Comput Assist Tomogr 1985;9:835-838.

35. Cho MH, Kim WS, Cho ZH. CSF flow artifact reduction using cardiac cycle ordered phaseencoding method. Magn Reson Imaging 1990;8:395-405.

36. Stenger VA, Peltier S, Boada FE, Noll DC. 3D spiral cardiac/respiratory ordered fMRI data acquisition at 3 Tesla. Magn Reson Med 1999;41:983-991. 
37. Frank LR, Jung Y, Inati S, Tyszka JM, Wong EC. High efficiency, low distortion 3D diffusion tensor imaging with variable density spiral fast spin echoes (3D DW VDS RARE). NeuroImage 2010;49:1510-1523.

38. Engström M, Skare S. Diffusion-weighted 3D multislab echo planar imaging for high signalto-noise ratio efficiency and isotropic image resolution. Magn Reson Med. 2013; doi: $10.1002 / \mathrm{mrm} .24594$.

39. Mansfield P, Howseman AM, Ordidge RJ. Volumar imaging using NMR spin echoes: echovolumar imaging (EVI) at 0.1 T. J Phys E: Sci Instrum 1989;22:324-330.

40. Mansfield P, Harvey PR, Stehling MK. Echo-volumar imaging. Magnetic Resonance Materials in Physics, Biology and Medicine 1994;2:291-294.

41. Greitz D, Wirestam R, Franck A, Nordell B, Thomsen C, Stahlberg F. Pulsatile brain movement and associated hydrodynamics studied by magnetic resonance phase imaging. The Monro-Kellie doctrine revisited. Neuroradiology 1992;34:370-380.

42. Enzmann DR, Pelc NJ. Brain motion: measurement with phase-contrast MR imaging. Radiology 1992;185:653-660.

43. Poncelet BP, Wedeen VJ, Weisskoff RM, Cohen MS. Brain parenchyma motion: measurement with cine echo-planar MR imaging. Radiology 1992;185:645-651.

44. Wirestam R, Greitz D, Thomsen C, Brockstedt S, Olsson MB, Stahlberg F. Theoretical and experimental evaluation of phase-dispersion effects caused by brain motion in diffusion and perfusion MR imaging. J Magn Reson Imaging 1996;6:348-355.

45. Franck A, Greitz D, Nordell B, Stahlberg F. A theoretical study of amplitude modulation and time shifting in quantitative MR measurements of motion in brain tissue. Magn Reson Imaging 1993;11:739-747.

46. Wansapura JP, Holland SK, Dunn RS, Ball WSJ. NMR relaxation times in the human brain at 3.0 tesla. J Magn Reson Imaging 1999;9:531-538.

47. Stanisz GJ, Odrobina EE, Pun J, Escaravage M, Graham SJ, Bronskill MJ, Henkelman RM. T1, T2 relaxation and magnetization transfer in tissue at 3T. Magn Reson Med 2005;54:507-512.

48. Feinberg DA, Setsompop K. Ultra-fast MRI of the human brain with simultaneous multislice imaging. J Magn Reson 2013;229:90-100.

49. Frost R, Jezzard P, Porter DA, Tijssen RHN, Miller KL. Simultaneous multi-slab acquisition in 3D multi-slab diffusion-weighted readout-segmented echo-planar imaging. In: Proceedings of the 21st Annual Meeting of ISMRM, 2013 (abstract 3176). 
50. Gudbjartsson H, Patz S. The Rician distribution of noisy MRI data. Magn Reson Med 1995;34:910-914.

51. Parker DL, Yuan C, Blatter DD. MR angiography by multiple thin slab 3D acquisition. Magn Reson Med 1991;17:434-451.

52. Oshio K, Jolesz FA, Melki PS, Mulkern RV. T2-weighted thin-section imaging with the multislab three-dimensional RARE technique. J Magn Reson Imaging 1991;1:695-700.

53. Pauly J, Le Roux P, Nishimura D, Macovski A. Parameter relations for the Shinnar-Le Roux selective excitation pulse design algorithm [NMR imaging]. IEEE Trans Med Imaging 1991;10:53-65.

54. Griswold MA, Jakob PM, Heidemann RM, Nittka M, Jellus V, Wang J, Kiefer B, Haase A. Generalized autocalibrating partially parallel acquisitions (GRAPPA). Magn Reson Med 2002;47:1202-1210.

55. Haacke EM, Lindskog ED, Lin W. A fast, iterative, partial-Fourier technique capable of local phase recovery. Journal of Magnetic Resonance (1969) 1991;92:126-145.

56. Morelli JN, Runge VM, Feiweier T, Kirsch JE, Williams KW, Attenberger UI. Evaluation of a modified Stejskal-Tanner diffusion encoding scheme, permitting a marked reduction in TE, in diffusion-weighted imaging of stroke patients at 3 T. Invest Radiol 2010;45:29-35.

57. Smith SM. Fast robust automated brain extraction. Hum Brain Mapp 2002;17:143-155.

58. Jenkinson M, Beckmann CF, Behrens TEJ, Woolrich MW, Smith SM. FSL. Neuroimage 2012;62:782-790.

59. Behrens TEJ, Berg HJ, Jbabdi S, Rushworth MFS, Woolrich MW. Probabilistic diffusion tractography with multiple fibre orientations: What can we gain? Neuroimage 2007;34:144-155.

60. Larkman DJ, Hajnal JV, Herlihy AH, Coutts GA, Young IR, Ehnholm G. Use of multicoil arrays for separation of signal from multiple slices simultaneously excited. J Magn Reson Imaging 2001;13:313-317.

61. Setsompop K, Gagoski BA, Polimeni JR, Witzel T, Wedeen VJ, Wald LL. Blippedcontrolled aliasing in parallel imaging for simultaneous multislice echo planar imaging with reduced g-factor penalty. Magn Reson Med 2012;67:1210-1224.

62. Jeurissen B, Leemans A, Tournier J, Jones DK, Sijbers J. Investigating the prevalence of complex fiber configurations in white matter tissue with diffusion magnetic resonance imaging. Hum Brain Mapp 2012; doi: 10.1002/hbm.22099. 


\section{Figures}

Figure 1: (a) Illustration of 2D and 3D k-space acquisition schemes in a 3D version of rsEPI. In each of the four panels, the dotted line represents the boundaries of the complete kspace matrix and the dot signifies the centre of 3D k-space. Solid lines represent acquisitions of one shot of k-space data. In the 2D versions ( $\mathrm{IM}_{2 \mathrm{D}}$ and $\left.\mathrm{NAV}_{2 \mathrm{D}}\right)$, these shots are simply $\mathrm{k}_{\mathrm{z}}$ phase-encoded readout segments. In the 3D versions ( $\mathrm{IM}_{3 \mathrm{D}}$ and $\mathrm{NAV}_{3 \mathrm{D}}$ ), each shot is a stack of readout segments with reduced $\mathrm{k}_{\mathrm{y}}$ extent, which is implemented in the pulse sequence by inserting $\mathrm{G}_{\mathrm{z}}$ blip gradients to move to the next $\mathrm{k}_{\mathrm{z}}$ plane and $\mathrm{G}_{\mathrm{y}}$ rewind gradients to move to the $\mathrm{k}_{\mathrm{y}}$ starting position. The low $\mathrm{k}_{\mathrm{y}}$ resolution readout segments are contiguous in $\mathrm{k}_{\mathrm{z}}$. For the imaging data ( $\mathrm{IM}_{2 \mathrm{D}}$ and $\left.\mathrm{IM}_{3 \mathrm{D}}\right)$, pre-phasing is used to move these shots around to sample the whole 3D k-space matrix. The navigator always acquires a full $\mathrm{k}_{\mathrm{y}}$ resolution readout segment $\left(\mathrm{NAV}_{2 \mathrm{D}}\right)$ or stack of low $\mathrm{k}_{\mathrm{y}}$ resolution readout segments $\left(\mathrm{NAV}_{3 \mathrm{D}}\right)$ at the centre of $3 \mathrm{D}$ k-space. (b) $\mathrm{IM}_{2 \mathrm{D}}+\mathrm{NAV}_{2 \mathrm{D}} 3 \mathrm{D}$ rs-EPI pulse sequence with modifications to the original rs-EPI sequence coloured red. Slab-selective RF pulses were used and $\mathrm{k}_{\mathrm{z}}$ phase-encoding was added. The $\mathrm{k}$-space shot was determined based on the position in the cardiac cycle so the kx and kz pre- and re-phasing gradients were updated in real-time. (c) Illustration with $8 \mathrm{k}_{\mathrm{z}}$ phase-encodes how adjacent slabs were overlapped and slices at either end of the slab were discarded. Also, the $\mathrm{k}_{\mathrm{z}}$ phase-encode FOV was oversampled to be larger than the slab profile defined by the full width at half maximum (FWHM) of the RF pulse.

Figure 2: Variation of phase and amplitude during one readout (left-hand column) and the resulting phase and amplitude variation in $\mathrm{k}_{\mathrm{y}}$ and $\mathrm{k}_{\mathrm{z}}$ during one shot of a IM $\mathrm{IMD}_{3 \mathrm{D}}$ trajectory (right-hand column). In the $\mathrm{k}_{\mathrm{y}}, \mathrm{k}_{\mathrm{z}}$ plots in the right-hand column, each $\mathrm{k}_{\mathrm{y}}$ point represents a $\mathrm{k}_{\mathrm{y}}$ phase-encode line of $\mathrm{k}_{\mathrm{x}}$ points. The numbers on the $\mathrm{k}_{\mathrm{y}}, \mathrm{k}_{\mathrm{z}}$ axes are counters of the line and partition, respectively. The amplitude of the image space mean signal was simulated at the central k-space point in a $120 \times 120 \times 10$ matrix using an in vivo field map to generate 
dephasing. The deviation of the shape of the amplitude curve from an ideal spin-echo is due to the dephasing from field inhomogeneity on top of the spin-echo formation. An average value of inhomogeneity was chosen to calculate a maximum phase error of $\pi / 2$ in the readout of $24 \times 24 \times 5$ points $\left(\mathrm{k}_{\mathrm{x}} \times \mathrm{k}_{\mathrm{y}} \times \mathrm{k}_{\mathrm{z}}\right)$ using a dwell time of $2 \mu \mathrm{s}$.

Figure 3: (a) $I M_{2 D}$ and (b) $I_{3 D}$ scheme simulation results. The modulation in amplitude and the associated point spread functions are shown in the $\mathrm{x}-\mathrm{y}$ and $\mathrm{y}-\mathrm{z}$ planes. In the $\mathrm{k}_{\mathrm{y}}, \mathrm{k}_{\mathrm{z}}$ plot, each $\mathrm{k}_{\mathrm{y}}$ point represents a $\mathrm{k}_{\mathrm{y}}$ phase-encode line of $\mathrm{k}_{\mathrm{x}}$ points. The scales on the amplitude and PSF plots are arbitrary.

Figure 4: (a) Images of the modified Shepp-Logan phantom (with added Rician noise) used in motion-induced phase simulations. The blue box indicates the slice being compared in part (b). (b) Simulation results for 8 and $24 \mathrm{k}_{\mathrm{z}}$ phase-encodes (16 and $48 \mathrm{~mm}$ slab thicknesses, respectively) with $b=1000 \mathrm{~s} / \mathrm{mm}^{2}$ diffusion weighting in $\mathrm{z}$. The acquisition and reconstruction approaches compared are no correction, 2D navigation with $\mathrm{NAV}_{2 \mathrm{D}}$ data, 3D navigation with $\mathrm{NAV}_{3 \mathrm{D}}$ data (all without re-acquisition) and 2D navigation with reacquisition, with and without cardiac reordering of shots. The coefficient of variation (CoV) in each voxel is calculated from the temporal standard deviation divided by the temporal mean over 6 repeated acquisition simulations. The repeated acquisitions have different motion phase corruption due to random variation of the timing of shots relative to the cardiac cycle. CoV maps were masked to show the voxels inside the phantom. (c) Simulation results showing a comparison of the mean and standard deviation of the coefficient of variation ( $\mathrm{CoV})$ for the different reconstruction approaches for 8 and $24 \mathrm{kz}$ phase-encodes with $\mathrm{b}=1000 \mathrm{~s} / \mathrm{mm}^{2}$ diffusion weighting in $\mathrm{z}$. The error bars show the standard deviation of the CoV. The means and standard deviations of the CoV reported are across the voxels within the high pulsatility region (velocity at peak systole $>0.5 \mathrm{~mm} / \mathrm{s}$ ).

Figure 5: (a) Experimental results showing a comparison of images and variability between sequential acquisition of k-space and cardiac-reordered acquisition of k-space in data with 8 and $16 \mathrm{k}_{\mathrm{z}}$ phase-encodes (left- and right-hand columns, respectively). Diffusion weighting is in the $\mathrm{z}$ direction with $\mathrm{b}=1000 \mathrm{~s} / \mathrm{mm} 2$ and the resolution of the images is $1.83 \times 1.83 \times 2$ 
$\mathrm{mm}^{2}$. The coefficient of variation $(\mathrm{CoV})$ in each voxel is calculated from the temporal standard deviation divided by the temporal mean over 6 repeated acquisitions in the data with 2D correction. (b) Locations of the ROIs used for quantitative comparisons of the coefficient of variation $(\mathrm{CoV})$ in the in vivo data with 8 and $16 \mathrm{k}_{\mathrm{z}}$ phase-encodes $(2 \mathrm{~mm}$ thick slices). The ROIs are shown on the diffusion-weighted images (all slices in the slab) with cardiac reordering and 2D correction. The outer slices have lower signal due to the reduced excitation at the edge of the slab profile.

Figure 6: Experimental results of a multi-slab DTI acquisition with $1.1 \mathrm{~mm}$ isotropic resolution and $\mathrm{b}=1000 \mathrm{~s} / \mathrm{mm}^{2}$ diffusion-weighting. (a) $\mathrm{T}_{2}$ - (b) diffusion-weighted (c) fractional anisotropy and (d) mean diffusivity images are shown.

Figure 7: Experimental results of the $1.3 \mathrm{~mm}$ isotropic multi-slab DTI data demonstrating the resolution. (a) Colour-coded maps of the principal eigenvector are shown along with zoomed regions overlaid on fractional anisotropy (FA) maps showing (a) cerebellar white matter projections and (b) cortical anisotropy. Note that in part (b) the FA map is inverted to show the cortex as bright. Colour code: green=A-P; blue=S-I; red=L-R.

Figure 8: Experimental results of the $1.3 \mathrm{~mm}$ isotropic multi-slab DTI data showing crossing fibres. (a) Mask (blue) indicating vowels in which BEDPOSTX fitting supported a second fibre population (at a threshold of a volume fraction >0.05). (b) Colour-coded map of the principal eigenvector with inset showing the corresponding vowel-wise vectors in a region of parietal white matter. The zoomed region demonstrates populations of fibres projecting to/from parietal cortex (blue, running S-I) which cross both callosal fibres at the bottom left (red, running R-L) and then the superior longitudinal fasciculus toward the top of the inset (green, running A-P).

Supplementary Figure 1: Plots of the component of brain tissue velocity in the $\mathrm{z}$ direction using the model described by Equations 1 and 2 from Ref. (43): temporal velocity variation at $\mathrm{x}=\mathrm{y}=\mathrm{z}=0$ with $\mathrm{t}_{0}=0.2 \mathrm{~s}$; velocity profile in $\mathrm{z}$ at $\mathrm{x}=\mathrm{y}=0$ and $\mathrm{t}=\mathrm{t}_{0}=0.2 \mathrm{~s}$; velocity profile in $\mathrm{x}$ at $\mathrm{y}=0, \mathrm{z}=40 \mathrm{~mm}$ and $\mathrm{t}=\mathrm{t}_{0}=0.2 \mathrm{~s}$. 
Supplementary Figure 2: An approximation of the SNR efficiency (signal $/ \sqrt{T R}$ ) as a function of TR in a spin-echo sequence. The spin-echo signal was calculated by numerical simulation of the Bloch equation with $\mathrm{TE}=70 \mathrm{~ms}$ and white matter values of $\mathrm{T}_{1}=1000 \mathrm{~ms}$ and $\mathrm{T}_{2}=75$ ms.

Supplementary Figure 3: Upper: the slice profile generated from the mean of all the diffusion images is plotted in green. The red plot shows an ideal, smooth variation of signal in the slice direction. An average slab profile was estimated from all slabs and a least squares fit for the slab amplitude was performed for the slice profile in each slab. The fits of all slabs to the slice profile are shown in blue. Lower: a correction factor for the slices at each slab boundary is shown in blue. The correction is generated from the signal loss between slabs in the fitted slice profile.

Supplementary Figure 4: The $1.1 \mathrm{~mm}$ isotropic data shown in Figure 6 without slab-join correction is provided for reference. Experimental results of a multi-slab DTI acquisition with $1.1 \mathrm{~mm}$ isotropic resolution and $\mathrm{b}=1000 \mathrm{~s} / \mathrm{mm}^{2}$ diffusion-weighting. (a) $\mathrm{T}_{2}$ - (b) diffusion-weighted (c) fractional anisotropy and (d) mean diffusivity images are shown.

Supplementary Figure 5: A version of Figure 7 generated with $1.1 \mathrm{~mm}$ isotropic data rather than $1.3 \mathrm{~mm}$ isotropic data. (a) Colour-coded maps of the principal eigenvector are shown along with zoomed regions overlaid on fractional anisotropy (FA) maps showing (a) cerebellar white matter projections and (b) cortical anisotropy. Note that in part (b) the FA map is inverted to show the cortex as bright. Colour code: green=A-P; blue=S-I; red=L-R. 


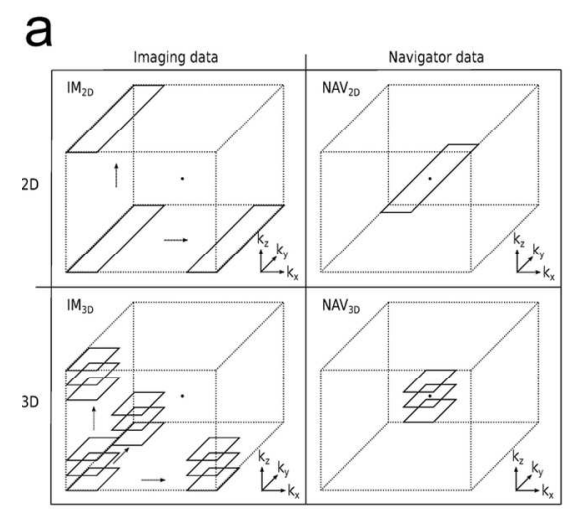

b
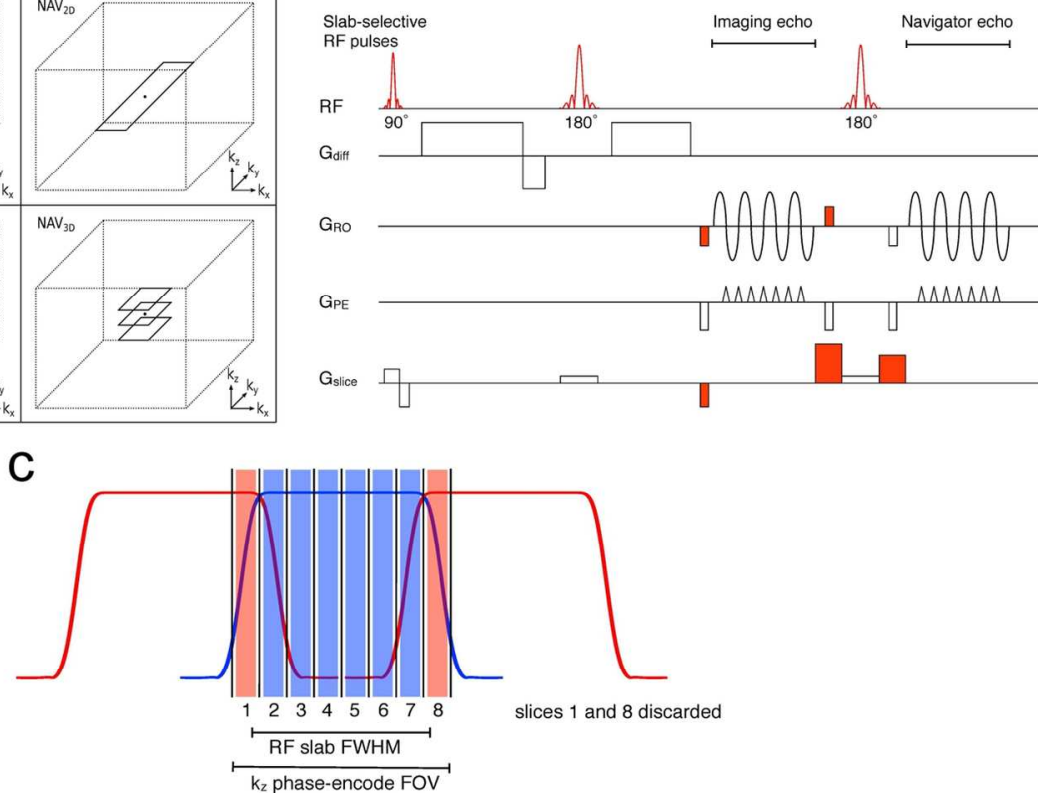

Figure 1: (a) Illustration of 2D and 3D k-space acquisition schemes in a 3D version of rs-EPI. In each of the four panels, the dotted line represents the boundaries of the complete k-space matrix and the dot signifies the centre of 3D k-space. Solid lines represent acquisitions of one shot of k-space data. In the 2D versions (IM2D and NAV2D), these shots are simply kz phase-encoded readout segments. In the 3D versions (IM3D and NAV3D), each shot is a stack of kx, ky planes, which is implemented in the pulse sequence by inserting Gz blip gradients to move to the next kz plane and Gy rewind gradients to move to the ky starting position. The kz planes are at contiguous kz points. For the imaging data (IM2D and IM3D), pre-phasing is used to move these shots around to sample the whole 3D k-space matrix. The navigator always acquires a full ky resolution readout segment (NAV2D) or stack of low ky resolution readout segments (NAV3D) at the centre of 3D k-space. (b) 3D rs-EPI pulse sequence with modifications to the original rs-EPI sequence coloured red. Slab-selective RF pulses were used and kz phase-encoding was added. The k-space shot was determined based on the position in the cardiac cycle so the kx and kz pre- and re-phasing gradients were updated in real-time. (c) Illustration with $8 \mathrm{kz}$ phase-encodes how adjacent slabs were overlapped and slices at either end of the slab were discarded. Also, the kz phase-encode FOV was oversampled to be larger than the slab profile defined by the full width at half maximum (FWHM) of the RF pulse.

$134 \times 89 \mathrm{~mm}(300 \times 300$ DPI $)$ 

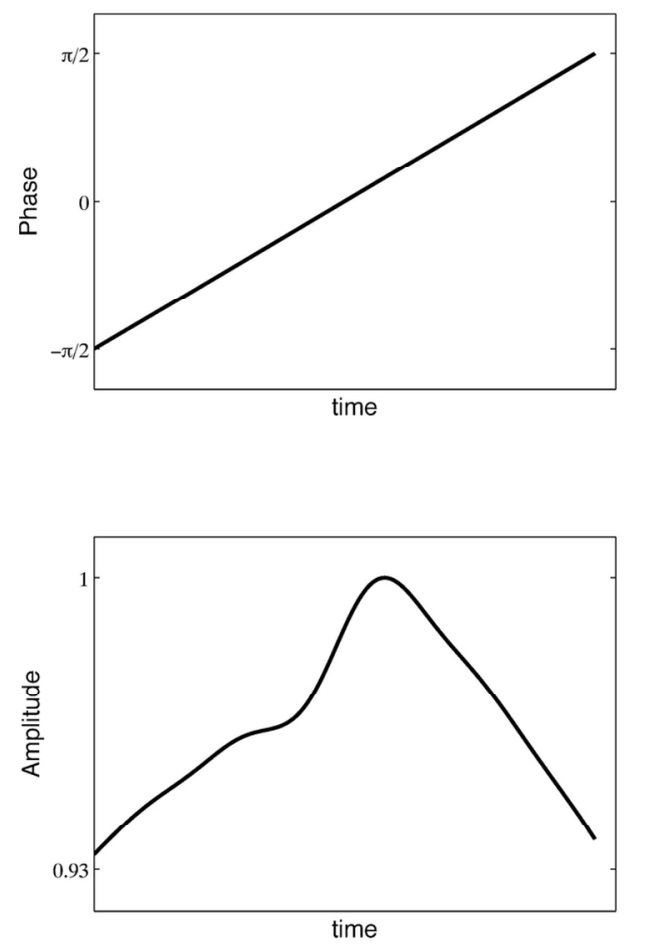

phase and amplitude during a spin-echo readout mapped onto one shot of an $\mathrm{IM}_{3 \mathrm{D}}$ trajectory
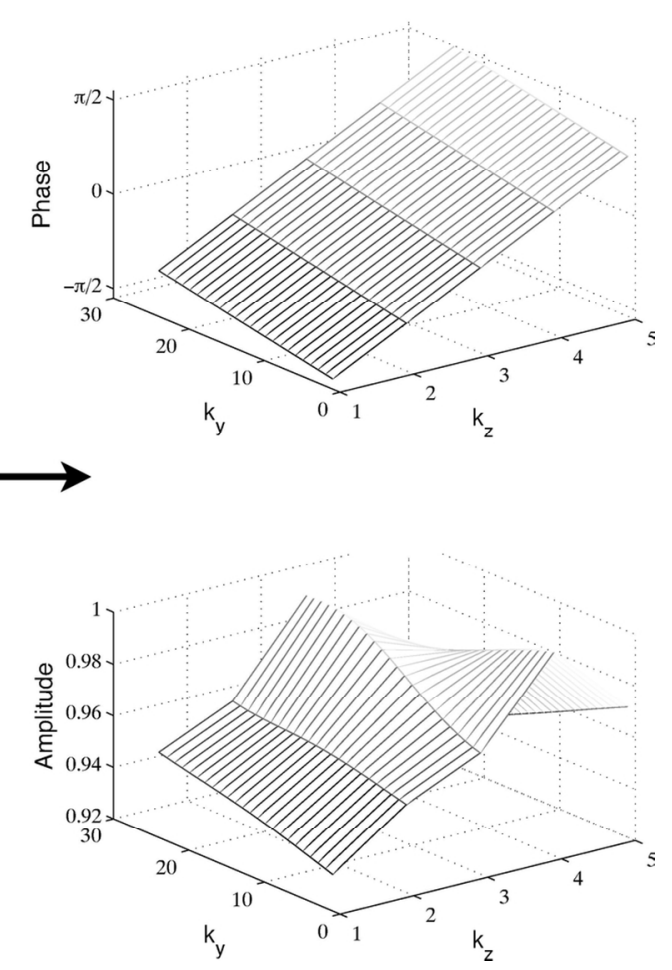

Figure 2: Variation of phase and amplitude during one readout (left-hand column) and the resulting phase and amplitude variation in ky and kz during one shot of a IM3D trajectory (right-hand column). In the ky, kz plots in the right-hand column, each ky point represents a ky phase-encode line of kx points. The numbers on the ky, kz axes are counters of the line and partition, respectively. The amplitude of the image space mean signal was simulated at the central $\mathrm{k}$-space point in a $120 \times 120 \times 10$ matrix using an in vivo field map to generate dephasing. The deviation of the shape of the amplitude curve from an ideal spin-echo is due to the dephasing from field inhomogeneity on top of the spin-echo formation. An average value of inhomogeneity was chosen to calculate a maximum phase error of $n / 2$ in the readout of $24 \times 24 \times 5$ points ( $k x \times k y \times k z)$ using a dwell time of $2 \mu s$. $119 \times 96 \mathrm{~mm}(300 \times 300 \mathrm{DPI})$ 
a
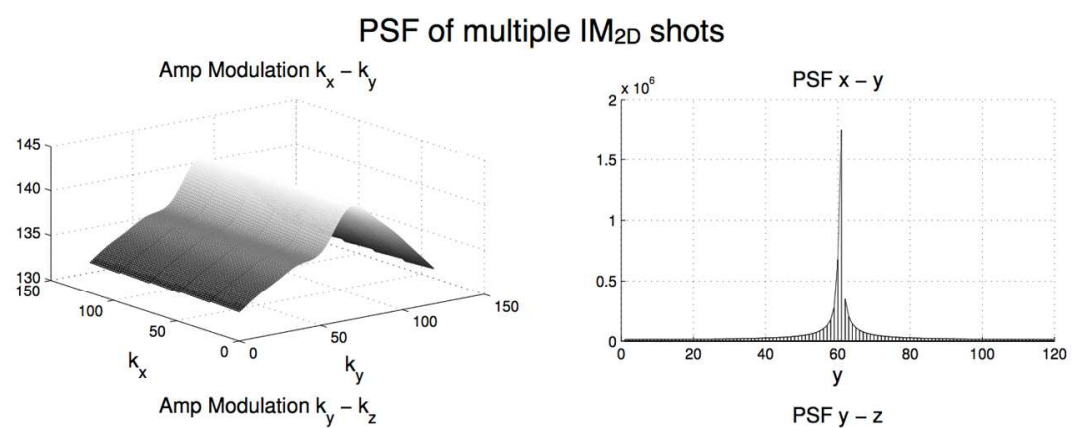

$\mathrm{k}_{\mathrm{z}}=0$ partition
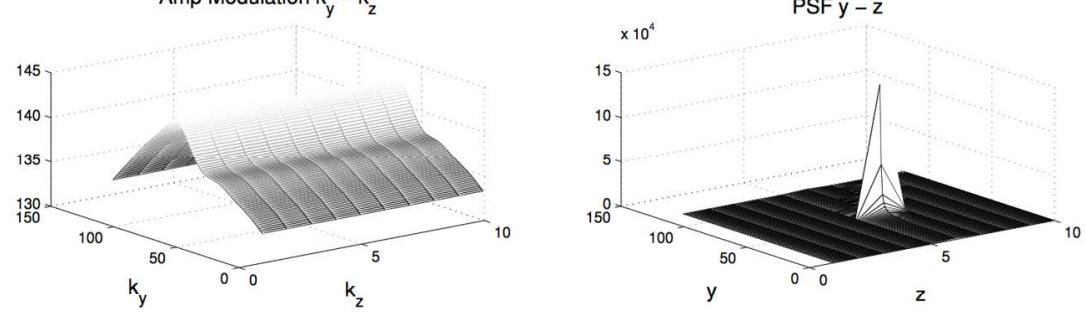

b

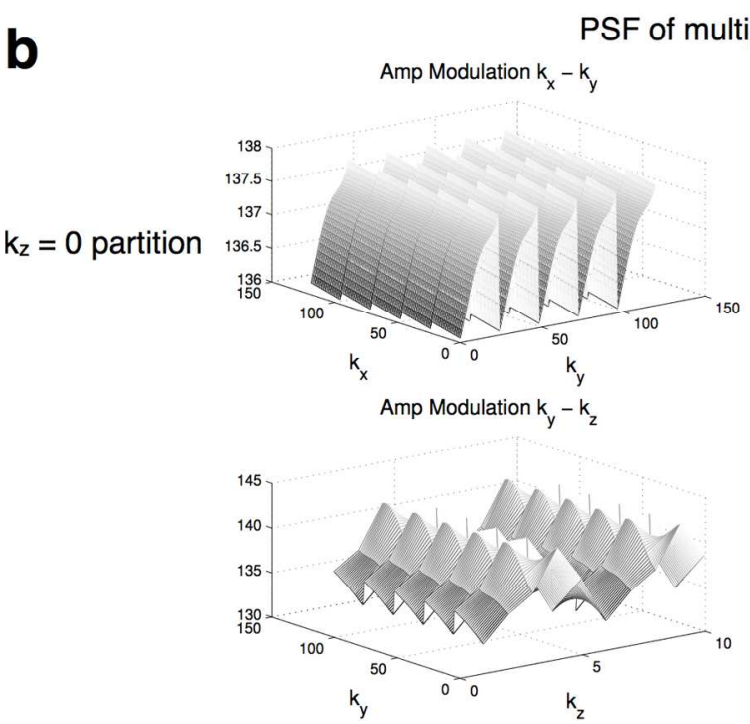

PSF of multiple $I M_{3 D}$ shots
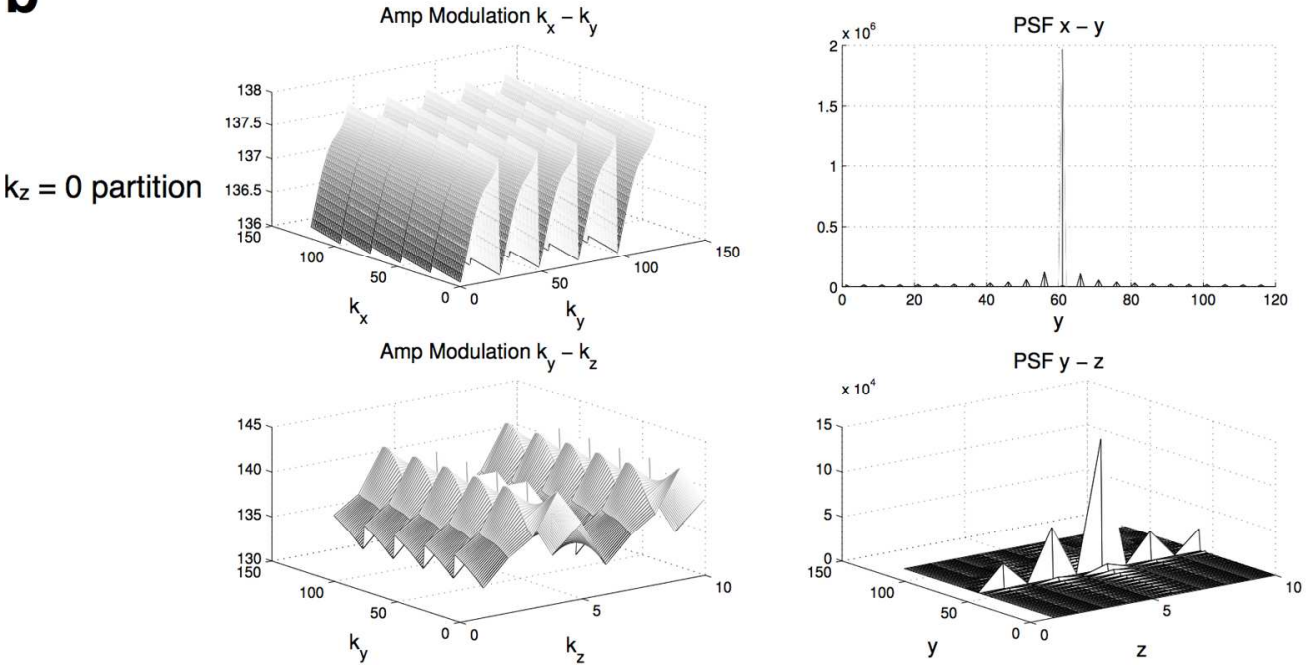

Figure 3: (a) IM2D and (b) IM3D scheme simulation results. The modulation in amplitude and the associated point spread functions are shown in the $x-y$ and $y$-z planes. In the ky, kz plot, each ky point represents a ky phase-encode line of $k x$ points. The scales on the amplitude and PSF plots are arbitrary. $146 \times 169 \mathrm{~mm}(300 \times 300 \mathrm{DPI})$ 
a

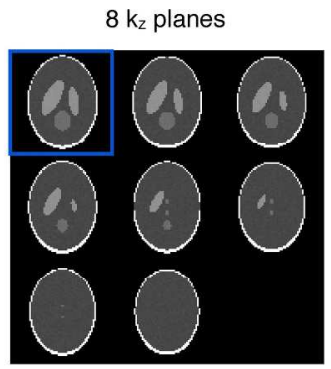

b
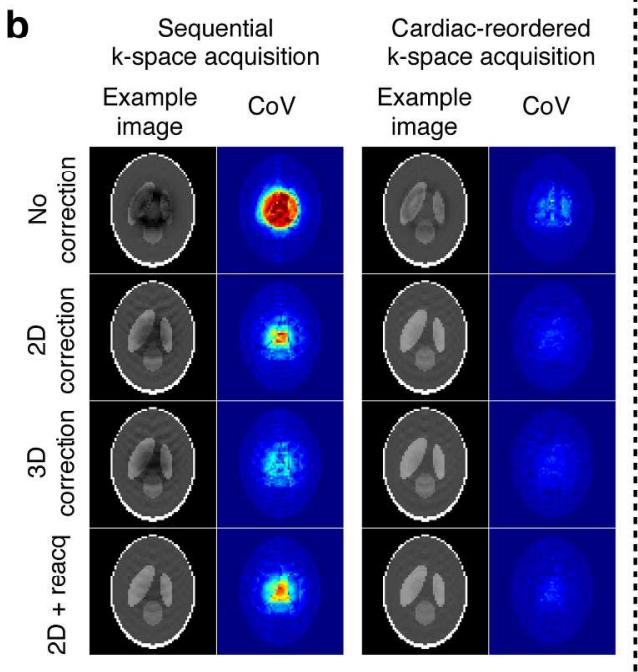

C

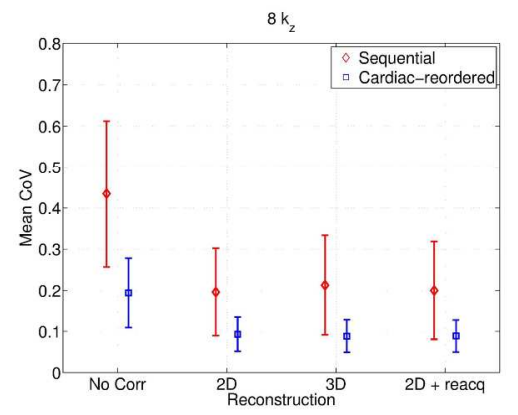

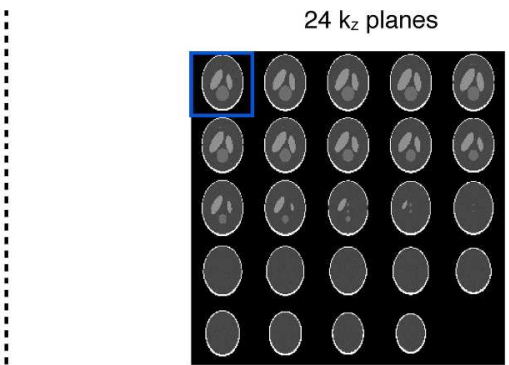
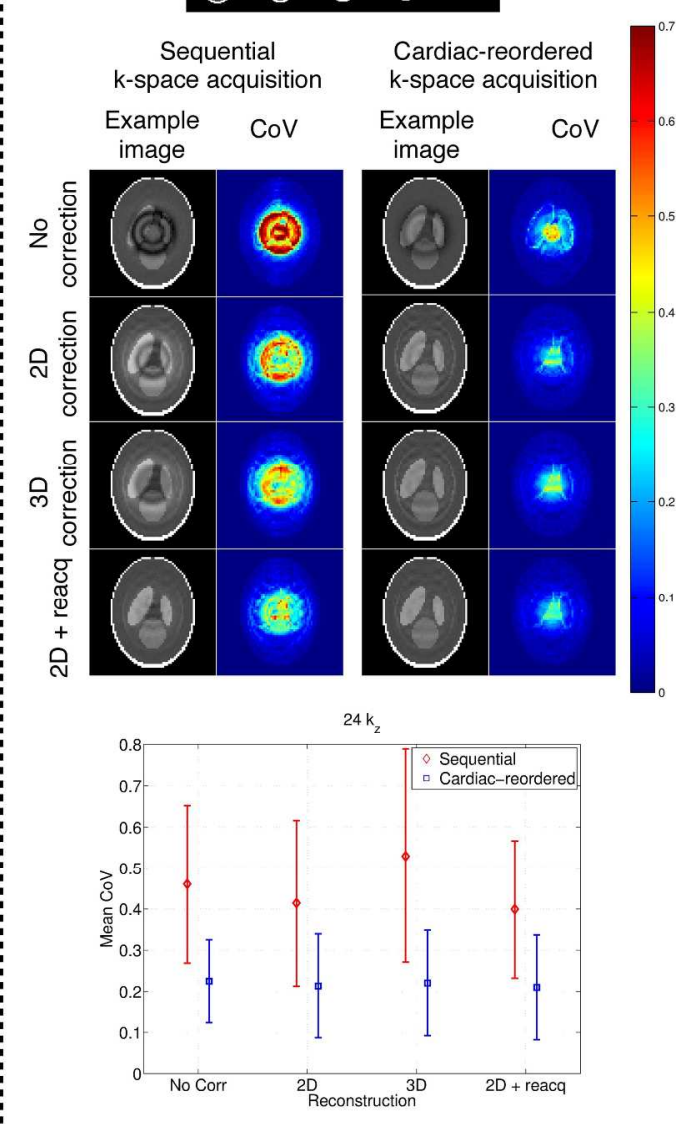

Figure 4: (a) Images of the modified Shepp-Logan phantom (with added Rician noise) used in motion-induced phase simulations. The blue box indicates the slice being compared in part (b). (b) Simulation results for 8 and $24 \mathrm{kz}$ phase-encodes (16 and $48 \mathrm{~mm}$ slab thicknesses, respectively) with $b=1000 \mathrm{~s} / \mathrm{mm} 2$ diffusion weighting in $z$. The acquisition and reconstruction approaches compared are no correction, 2D navigation with NAV2D data, 3D navigation with NAV3D data (all without re-acquisition) and 2D navigation with reacquisition, with and without cardiac reordering of shots. The coefficient of variation (CoV) in each voxel is calculated from the temporal standard deviation divided by the temporal mean over 6 repeated acquisition simulations. The repeated acquisitions have different motion phase corruption due to random variation of the timing of shots relative to the cardiac cycle. CoV maps were masked to show the voxels inside the phantom. (c) Simulation results showing a comparison of the mean and standard deviation of the coefficient of variation (CoV) for the different reconstruction approaches for 8 and $24 \mathrm{kz}$ phase-encodes with $b=1000 \mathrm{~s} / \mathrm{mm} 2$ diffusion weighting in $\mathrm{z}$. The error bars show the standard deviation of the CoV. The means and standard deviations of the CoV reported are across the voxels within the high pulsatility region (velocity at peak systole $>0.5 \mathrm{~mm} / \mathrm{s}$ ). 


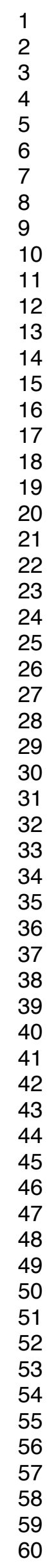

$233 \times 265 \mathrm{~mm}(300 \times 300 \mathrm{DPI})$

Magnetic Resonance in Medicine 

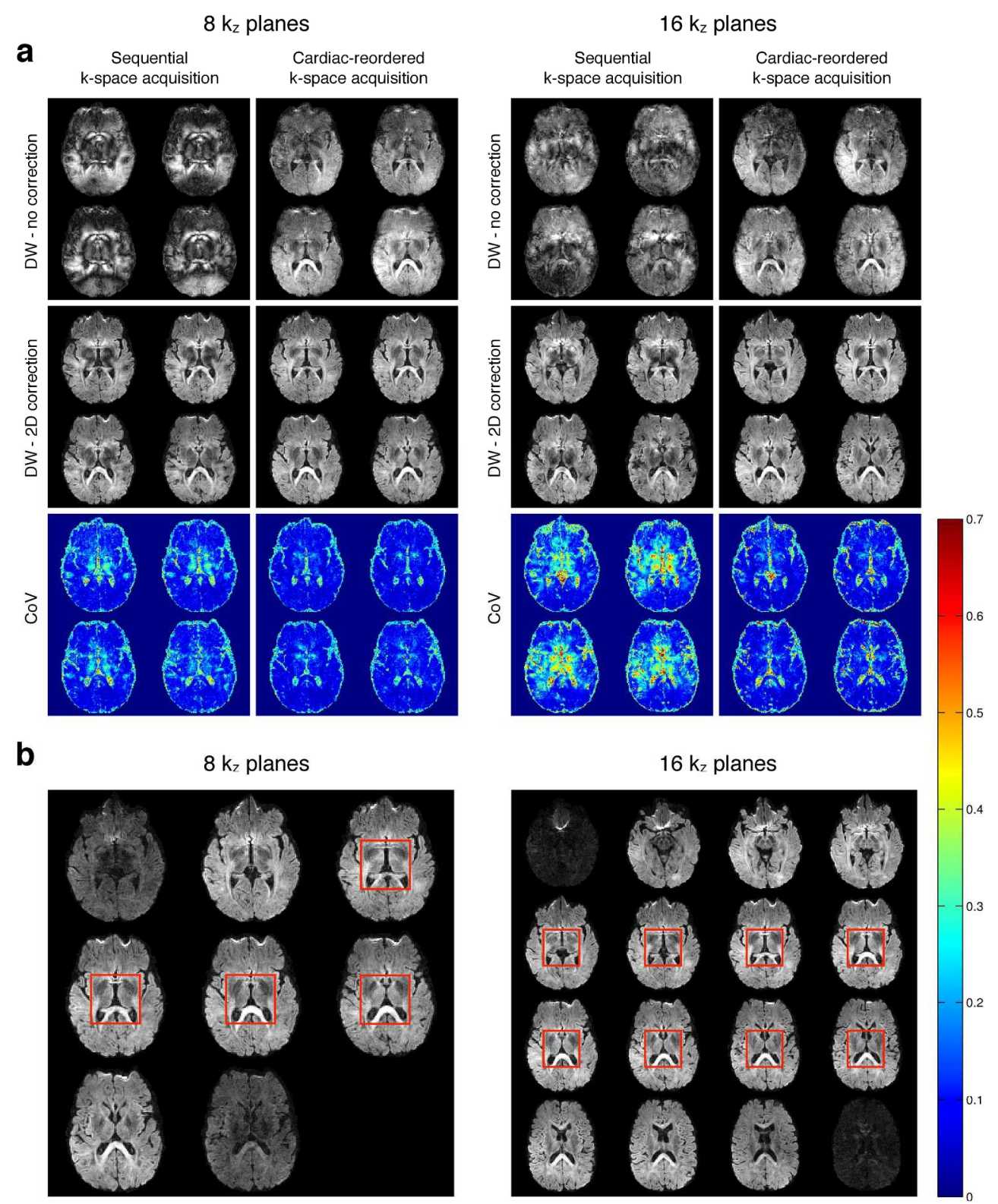

Figure 5: (a) Experimental results showing a comparison of images and variability between sequential acquisition of k-space and cardiac-reordered acquisition of k-space in data with 8 and $16 \mathrm{kz}$ phase-encodes (left- and right-hand columns, respectively). Diffusion weighting is in the $z$ direction with $b=1000 \mathrm{~s} / \mathrm{mm} 2$ and the resolution of the images is $1.83 \times 1.83 \times 2 \mathrm{~mm} 2$. The coefficient of variation (CoV) in each voxel is calculated from the temporal standard deviation divided by the temporal mean over 6 repeated acquisitions in the data with 2D correction. (b) Locations of the ROIs used for quantitative comparisons of the coefficient of variation (CoV) in the in vivo data with 8 and $16 \mathrm{kz}$ phase-encodes ( $2 \mathrm{~mm}$ thick slices). The ROIs are shown on the diffusion-weighted images (all slices in the slab) with cardiac reordering and 2D correction. The outer slices have lower signal due to the reduced excitation at the edge of the slab profile. $231 \times 284 \mathrm{~mm}(300 \times 300$ DPI) 

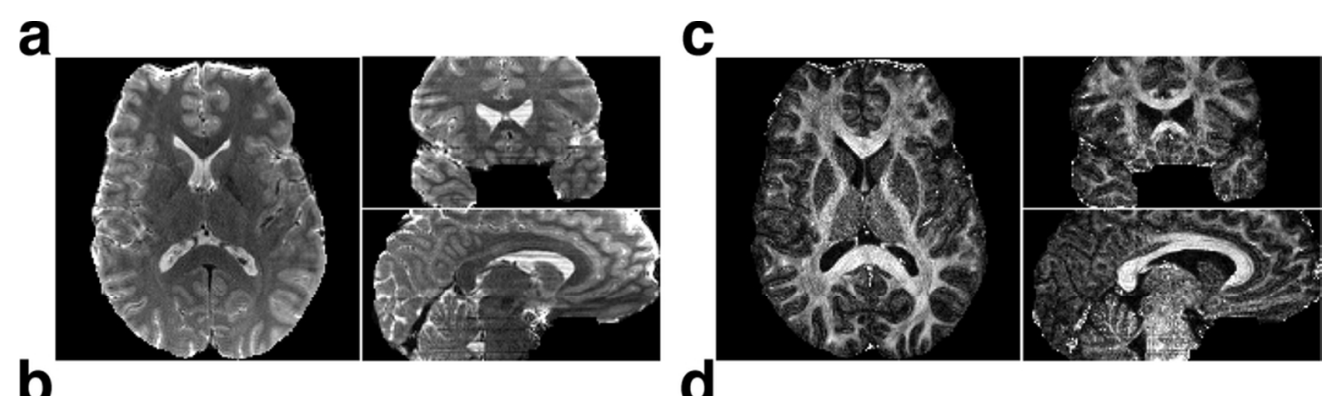

Figure 6: Experimental results of a multi-slab DTI acquisition with $1.1 \mathrm{~mm}$ isotropic resolution and $\mathrm{b}=1000$ s/mm2 diffusion-weighting. (a) T2- (b) diffusion-weighted (c) fractional anisotropy and (d) mean diffusivity images are shown. $90 \times 47 \mathrm{~mm}(300 \times 300 \mathrm{DPI})$ 

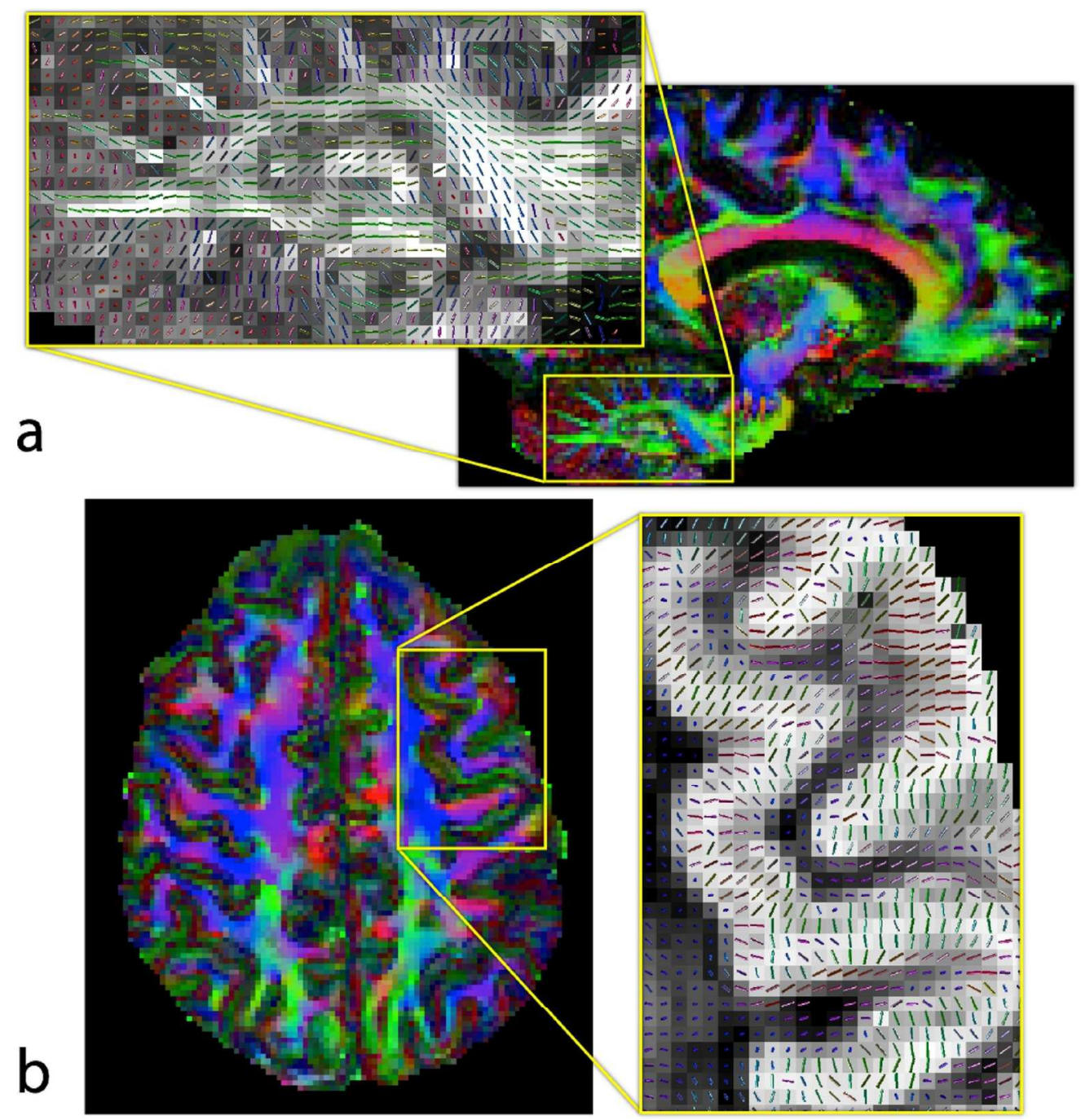

Figure 7: Experimental results of the $1.3 \mathrm{~mm}$ isotropic multi-slab DTI data demonstrating the resolution. (a) Colour-coded maps of the principal eigenvector are shown along with zoomed regions overlaid on fractional anisotropy (FA) maps showing (a) cerebellar white matter projections and (b) cortical anisotropy. Note that in part (b) the FA map is inverted to show the cortex as bright. Colour code: green=A-P; blue=S-I; red=L-R. $127 \times 133 \mathrm{~mm}(300 \times 300 \mathrm{DPI})$ 

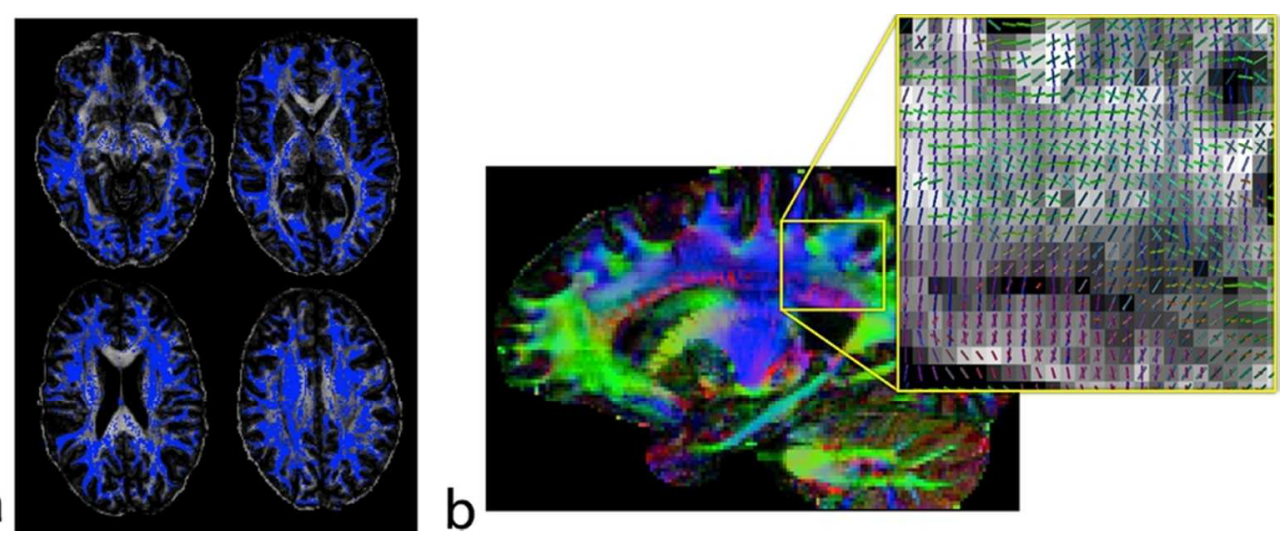

Figure 8: Experimental results of the $1.3 \mathrm{~mm}$ isotropic multi-slab DTI data showing crossing fibres. (a) Mask (blue) indicating vowels in which BEDPOSTX fitting supported a second fibre population (at a threshold of a volume fraction $>0.05$ ). (b) Colour-coded map of the principal eigenvector with inset showing the corresponding vowel-wise vectors in a region of parietal white matter. The zoomed region demonstrates populations of fibres projecting to/from parietal cortex (blue, running S-I) which cross both callosal fibres at the bottom left (red, running R-L) and then the superior longitudinal fasciculus toward the top of the inset (green, running A-P). $78 \times 31 \mathrm{~mm}(300 \times 300 \mathrm{DPI})$ 


\section{Acquisition Parameters}

\begin{tabular}{|c|c|c|c|c|c|c|c|c|c|c|c|}
\hline $\begin{array}{c}\text { Resolution } \\
(\mathrm{mm})\end{array}$ & $\begin{array}{l}\text { FOV } \\
(\mathrm{cm}) \\
\end{array}$ & Matrix & $\begin{array}{c}\text { Readout } \\
\text { segments }\end{array}$ & $\begin{array}{c}\mathrm{ES}^{*} \\
(\mathrm{~ms}) \\
\end{array}$ & $R_{P E}$ & $\begin{array}{c}\text { \# slabs / } \\
\text { slices per slab } \\
\end{array}$ & $\begin{array}{c}\text { Slab overlap / } \\
\text { discarded slices }\end{array}$ & $\begin{array}{c}\text { Odd/even } \\
\text { slab concat. }\end{array}$ & $\begin{array}{l}\alpha / \mathrm{TR} / \mathrm{TE} \\
(\mathrm{s} / \mathrm{s} / \mathrm{ms}) \\
\end{array}$ & $\begin{array}{c}\text { Exc. / refoc. } \\
\text { durations (ms) }\end{array}$ & $\begin{array}{l}\text { Scan time } \\
\text { (min) }\end{array}$ \\
\hline \multicolumn{12}{|c|}{ Repeated acquisition protocols: 6 volumes with $\mathrm{b}=1000 \mathrm{~s} / \mathrm{mm}^{2}$ weighting in $\mathrm{z}$ and $1 \mathrm{~b}=0$ image. } \\
\hline $1.83 \times 1.83 \times 2$ & $22 \times 22$ & $120 \times 120$ & 5 & 0.32 & - & $1 / 8$ & $-/-$ & - & $68 / 1 / 87$ & $2.5 / 5$ & $4: 45$ \\
\hline $1.83 \times 1.83 \times 2$ & $22 \times 22$ & $120 \times 120$ & 5 & 0.32 & - & $1 / 16$ & $-1-$ & - & $68 / 1 / 87$ & $2.5 / 5$ & $9: 25$ \\
\hline \multicolumn{12}{|c|}{ Multi-slab protocols: 20 diffusion directions with $\mathrm{b}=1000 \mathrm{~s} / \mathrm{mm}^{2}$ weighting and $3 \mathrm{~b}=0$ images. } \\
\hline & $22 \times 22$ & $170 \times 170$ & 5 & 0.38 & 2 & $16 / 8$ & & yes & 80 / 1.8 / 83 & $2.5 / 5$ & 55 \\
\hline 1.1 iso & $22 \times 22$ & $200 \times 200$ & $4(4 / 5 \mathrm{PF})$ & 0.40 & 2 & $14 / 10$ & $40 \% / 4$ & no & $90 / 3.9 / 87$ & $5 / 10$ & 60 \\
\hline
\end{tabular}

*The echo-spacing (ES) values are the times between successive echoes, rather than the effective echo-spacing after taking GRAPPA acceleration into account. 


\begin{tabular}{lc} 
Simulation & \\
\hline CoV $8 \mathrm{k}_{\mathrm{z}}$ Seq & $0.071 \pm 0.081$ \\
CoV $8 \mathrm{k}_{\mathrm{z}}$ CR & $0.044 \pm 0.036$ \\
$\%$ improvement & 38 \\
CoV $16 \mathrm{k}_{\mathrm{z}}$ Seq & $0.144 \pm 0.199$ \\
CoV $16 \mathrm{k}_{\mathrm{z}}$ CR & $0.059 \pm 0.065$ \\
$\%$ improvement & 59 \\
CoV $24 \mathrm{k}_{\mathrm{z}}$ Seq & $0.155 \pm 0.176$ \\
CoV $24 \mathrm{k}_{\mathrm{z}}$ CR & $0.075 \pm 0.083$ \\
$\%$ improvement & 52
\end{tabular}

\section{Experiment}

\begin{tabular}{lccccc}
\hline & Subj. 1 & Subj. 2 & Subj. 3 & Subj. 4 & Subj. 5 \\
\hline CoV 8 k Seq & $0.153 \pm 0.102$ & $0.174 \pm 0.105$ & $0.168 \pm 0.108$ & $0.243 \pm 0.128$ & $0.179 \pm 0.113$ \\
CoV 8 k CR & $0.098 \pm 0.068$ & $0.125 \pm 0.087$ & $0.121 \pm 0.092$ & $0.121 \pm 0.088$ & $0.145 \pm 0.091$ \\
$\%$ improvement & 36 & 28 & 28 & 50 & 19 \\
CoV 16 k Leq $_{\text {CoV 16 k }}$ CR & $0.236 \pm 0.150$ & $0.257 \pm 0.133$ & $0.200 \pm 0.141$ & $0.274 \pm 0.147$ & $0.274 \pm 0.129$ \\
\% improvement & $0.118 \pm 0.104$ & $0.160 \pm 0.124$ & $0.138 \pm 0.117$ & $0.163 \pm 0.119$ & $0.127 \pm 0.105$ \\
& 50 & 38 & 31 & 41 & 54
\end{tabular}

Table 1: Comparison of the variability in sequential and cardiac-reordered k-space acquisitions in simulations and experiment. The coefficient of variation $(\mathrm{CoV})$ in each voxel was calculated by dividing the temporal standard deviation by the temporal mean over 6 volumes of $\mathrm{b}=1000 \mathrm{~s} / \mathrm{mm}^{2}$ diffusion weighting in the $\mathrm{z}$ direction. The CoV reported is the mean within the ROIs defined by the high pulsatility region in the phantom and in Fig. $5 \mathrm{~b}$ (for the simulation and experimental results, respectively) and the error is the standard deviation within the ROI. Both the simulation and experiment results are when using the 2D navigator correction (without re-acquisition). 


\section{Page 73 of 78}

1

2

3

4

5

6

7

8

10

11

12

13

14

15

16

17

18

19

20

21

22

23

24

25

26

27

28

29

30

31

32

33

34

35

36

37

38

39

40

41

42

43

44

45

46

47

48

49

50

51

52

53

54

55

56

57

58

59

60

\section{Magnetic Resonance in Medicine}
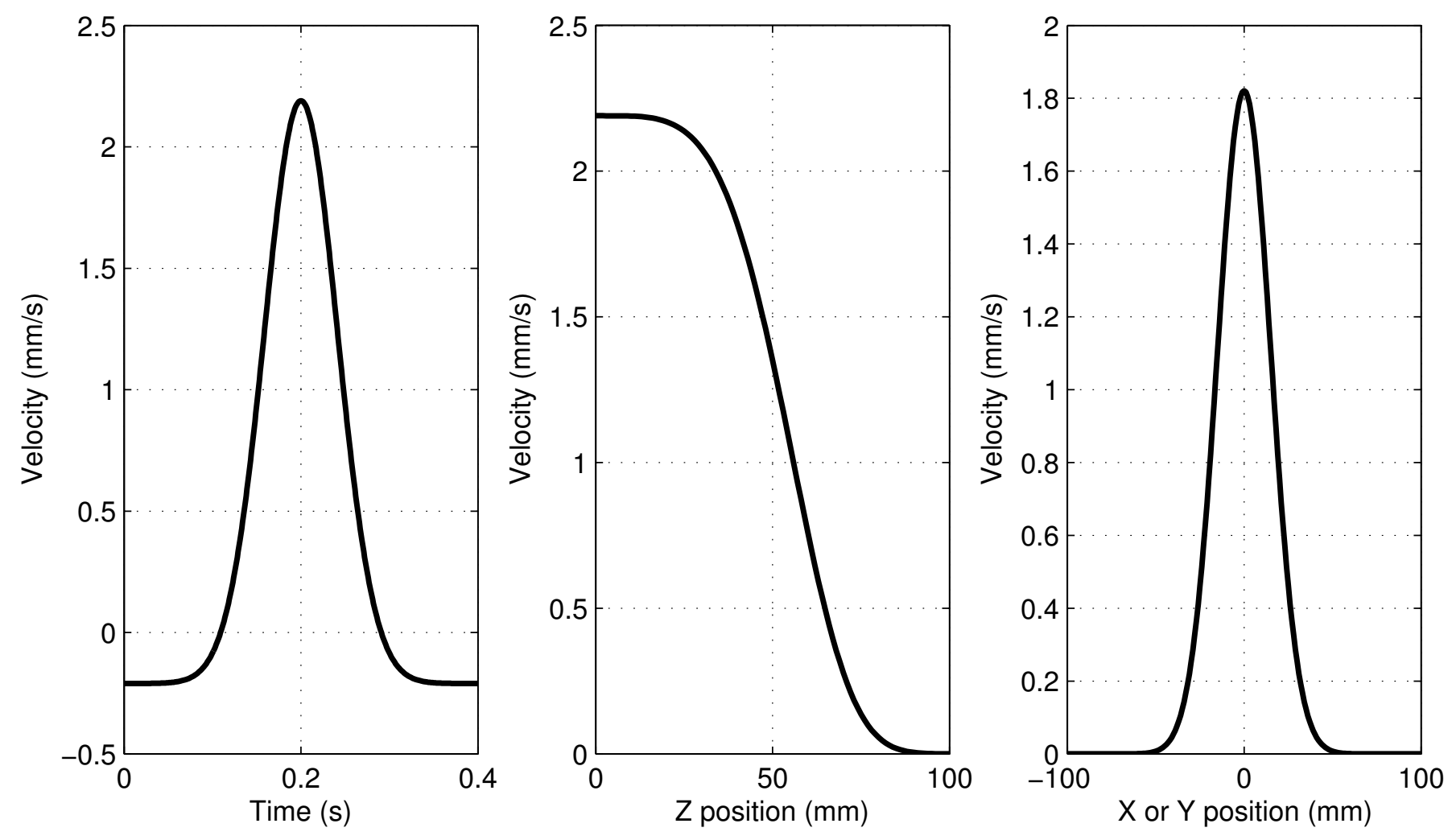


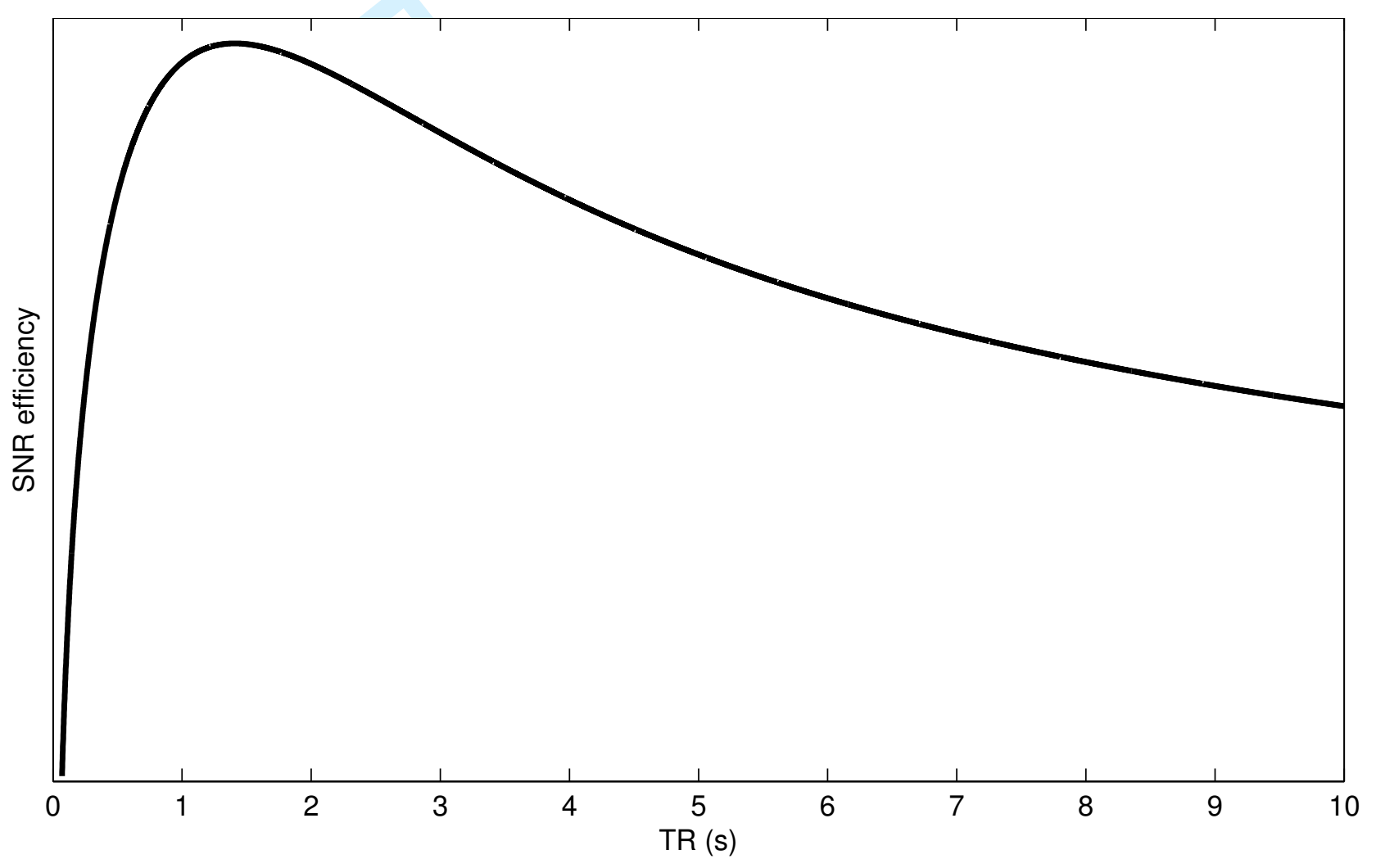

Magnetic Resonance in Medicine 
Signal profile and fit in mean DW image

施

$\frac{\mathrm{P}}{30} \mathrm{C}$

3

31
33
34
34

3

be

3
3
3
4
4
4
4
4
4

4
4
4
4

4
4
4
4

(1)

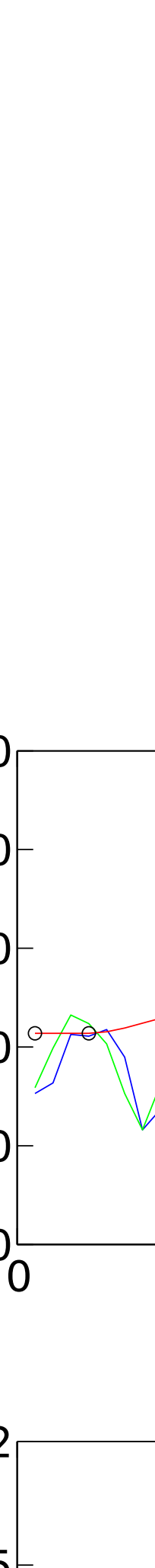

-

-

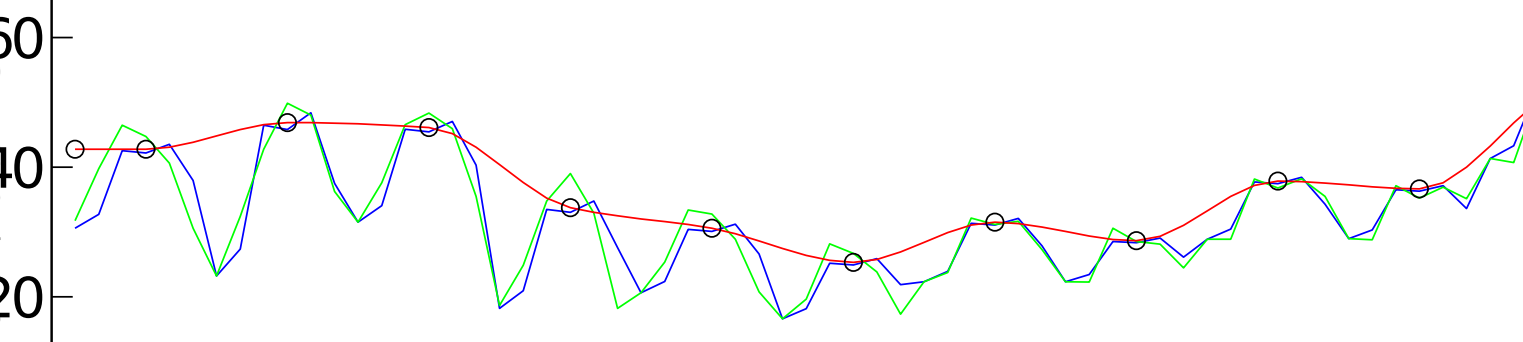

西

1

42

43 44

14
5
5
5
5
5
5
5
15

1
5
5
5
5
5
5
5
15
5
5
6

6

1

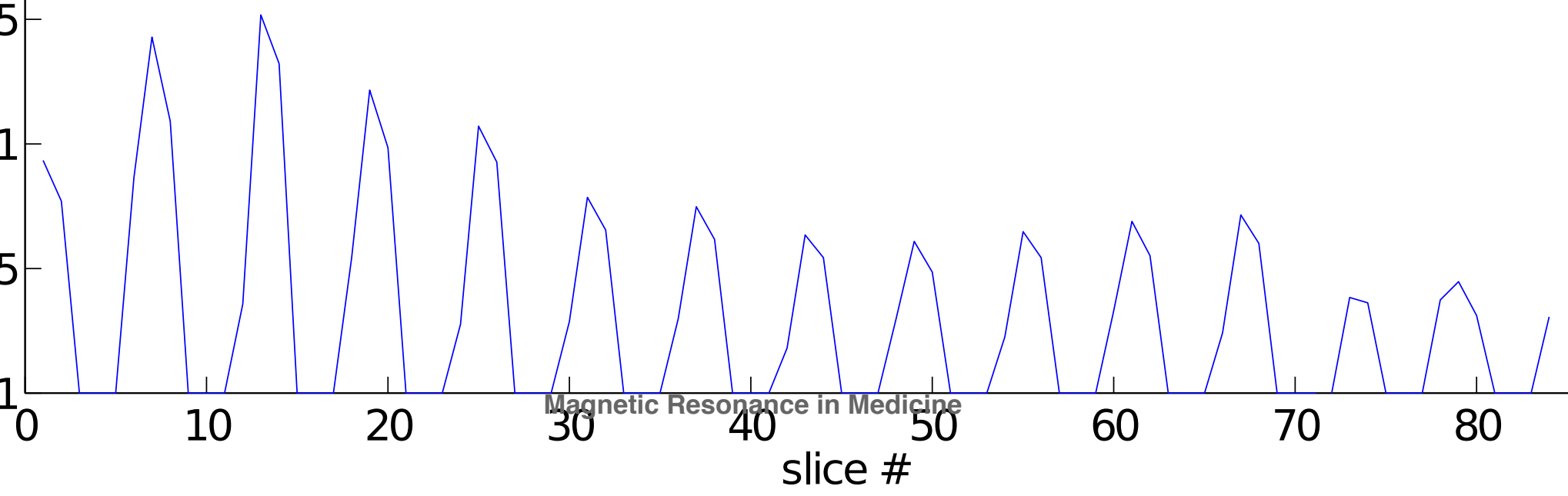


a
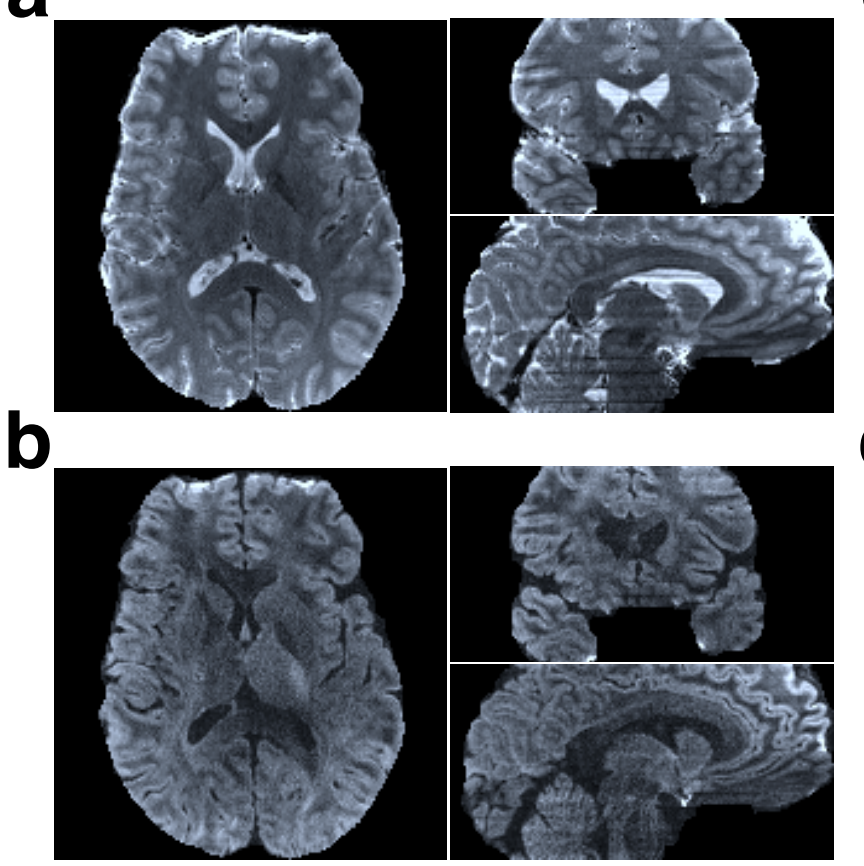

C

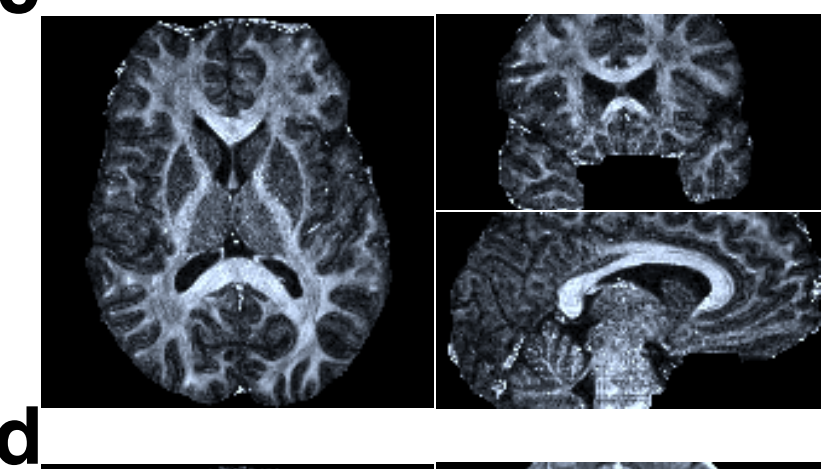

Magnetic Resonance in Medicine 


\section{Supplementary Algorithm}

This Appendix provides Matlab code of the algorithm used to choose which k-space shot to acquire based on the value of the cardiac phase, $\varphi_{c}$ ("phic" in the code). $\varphi_{c}$ was calculated by dividing the time since the cardiac trigger, $t_{\text {trig, }}$ measured with a pulse oximeter placed on the index finger (a 200 ms delay time was determined empirically), by the mean cardiac cycle duration, $t_{R R}$ (from the previous 10 cardiac cycles).

A cardiac phase of $0.4-0.6$ was assumed to correspond to diastole and therefore to be the optimum time to acquire data. The following algorithm was used to determine the $\mathrm{k}_{\mathrm{z}}$ and $\mathrm{k}_{\mathrm{x}}$ shot indices (" $\mathrm{nz}$ " and " $\mathrm{nx}$ ", respectively):

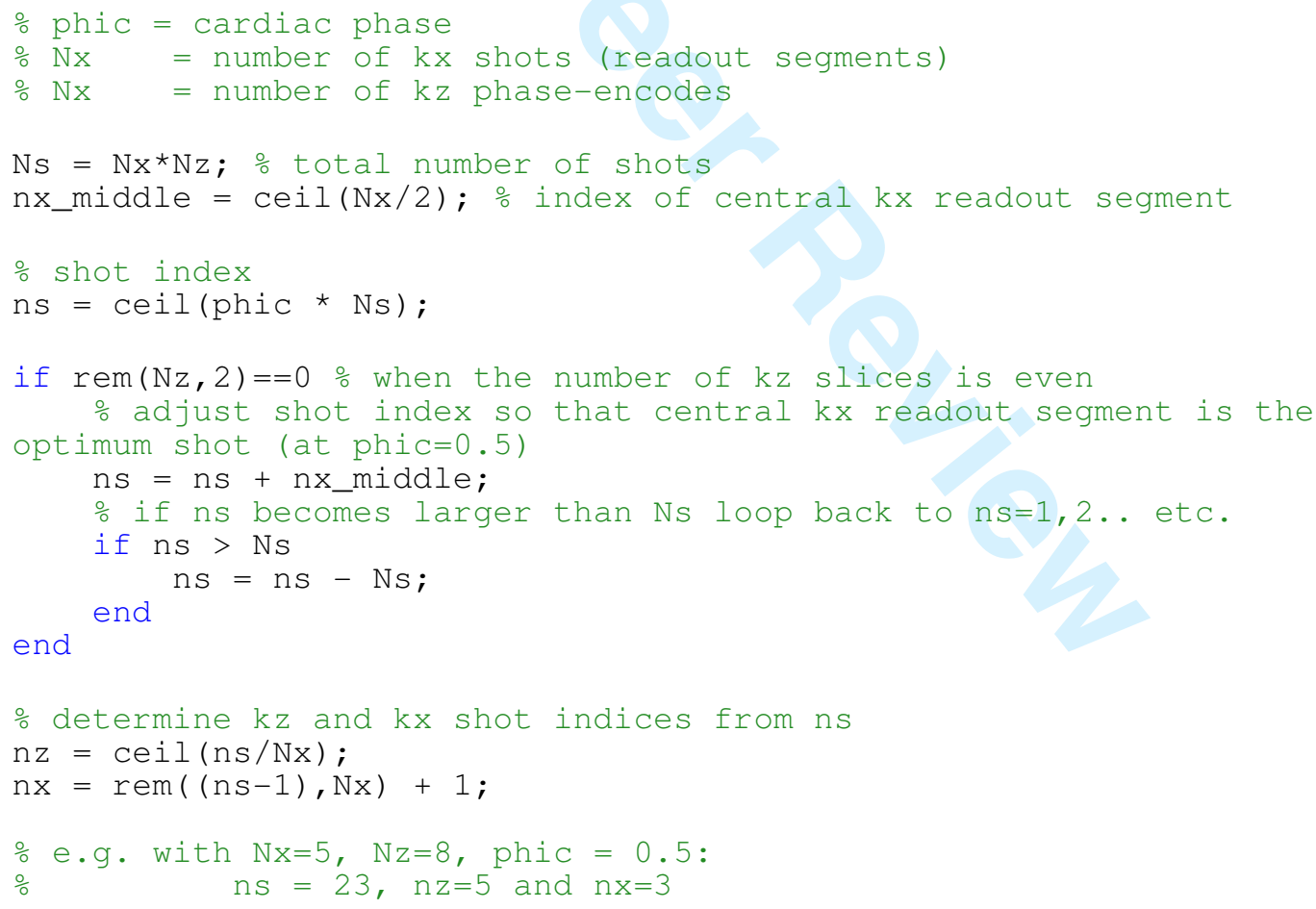

